\title{
Insights into leading edge vortex formation and detachment on a pitching and plunging flat plate
}

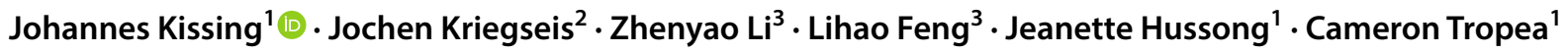

Received: 4 March 2020 / Revised: 24 July 2020 / Accepted: 13 August 2020 / Published online: 7 September 2020

(c) The Author(s) 2020

\begin{abstract}
The present study is a prelude to applying different flow control devices on pitching and plunging airfoils with the intention of controlling the growth of the leading edge vortex (LEV); hence, the lift under unsteady stall conditions. As a pre-requisite the parameters influencing the development of the LEV topology must be fully understood and this constitutes the main motivation of the present experimental investigation. The aims of this study are twofold. First, an approach is introduced to validate the comparability between flow fields and LEV characteristics of two different facilities using water and air as working media by making use of a common baseline case. The motivation behind this comparison is that with two facilities the overall parameter range can be significantly expanded. This comparison includes an overview of the respective parameter ranges, control of the airfoil kinematics and careful scrutiny of how post-processing procedures of velocity data from timeresolved particle image velocimetry (PIV) influence the integral properties and topological features used to characterise the LEV development. Second, and based on results coming from both facilities, the appearance of secondary structures and their effect on LEV detachment over an extended parameter range is studied. A Lagrangian flow field analysis based on finite-time Lyapunov Exponent (FTLE) ridges allows precise identification of secondary structures and reveals that their emergence is closely correlated to a vortex Reynolds number threshold computed from the LEV circulation. This threshold is used to model the temporal onset of secondary structures. Further analysis indicates that the emergence of secondary structures causes the LEV to stop accumulating circulation if the shear layer angle at the leading edge of the flat plate has ceased to increase. This information is of particular importance for advanced flow control applications, since efforts to strengthen and/ or prolong LEV growth rely on precise knowledge about where and when to apply flow control measures.
\end{abstract}

\section{Graphical abstract}

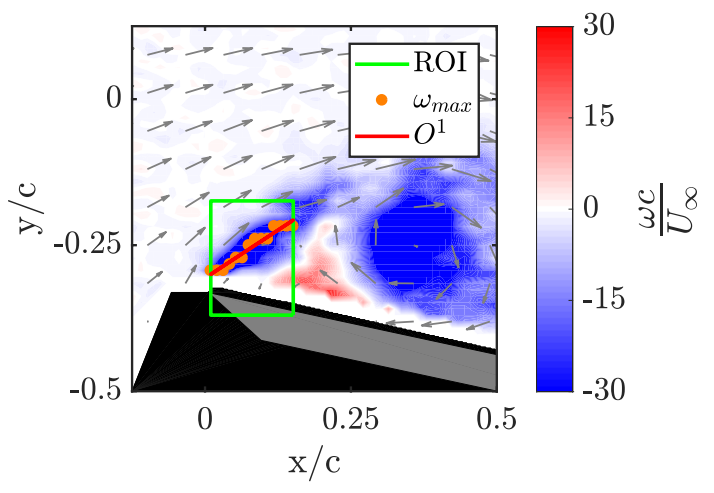

Johannes Kissing

kissing@sla.tu-darmstadt.de

Extended author information available on the last page of the article

\section{Introduction}

High lift at low Reynolds numbers is an essential feature of biological propulsion based on flapping wings and is a promising technology for future micro air vehicle (MAV). 
In terms of dimensionless parameters, hovering insects and birds in cruise flight were found to execute wing kinematics at a chord based Reynolds number $\operatorname{Re}=U_{\infty} c / v$ of the order of $10^{3}-10^{4}$, where $U_{\infty}$ is the free-stream velocity, $c$ the airfoil chord and $v$ is the kinematic viscosity (cf. Ellington 1984). The Strouhal number St $=2 f h / U_{\infty}$, where $f$ is the plunging frequency and $h$ the plunging amplitude, varies between 0.2 and 0.4 for efficient propulsion, while the reduced frequency $k=\pi c f / U_{\infty}$ is optimized according to the respective St and the wing planform area (cf. Triantafyllou et al. 1993; Nudds et al. 2004, etc.) MAVs are designed for a wide range of Re from 0 up to 60,000, a higher $\mathrm{k}$ between 0.146 and 1.2 and lower St up to 0.07, compared to biological flapping flight, due to the high inertial loads of moving wings (cf. Jones and Babinsky 2010; Jones and Platzer 2000; de Croon et al. 2015).

High transient lift on flapping wings of insects and birds is attributed to leading edge vortex (LEV) growth on the wing (e.g. Ellington et al. 1996). It occurs when the effective angle of attack of the inflow on the airfoil $\left(\alpha_{\text {eff }}\right)$ changes dynamically, such that the leading edge shear layer separates due to an adverse pressure gradient and subsequently rolls up into a vortex. This process, known as dynamic stall (cf. Carr 1988), leads to a collapse of the induced lift as soon as the LEV detaches from the airfoil and is convected downstream. Therefore, maintaining a longer vortex growth phase by delaying its detachment with the aid of local flow control can increase the overall vortex induced lift on flapping wings and thus enhance the manoeuvrability as well as the gust-tolerance of MAVs (cf. Eldredge and Jones 2019).

The long-term objective of the current study is manipulation of the flow field around the LEV on a pitching and/or plunging flat plate in order to attain higher overall transient lift by delaying the LEV detachment or increasing its circulation. The idea is to manipulate the flow field at topologically critical locations using a dielectric barrier discharge plasma actuator (DBD-PA) in air and a synthetic jet actuator (SJ) in water. To enable this manipulation the underlying mechanisms have to first be sufficiently understood.

Rival et al. (2014) found the chord length $c$ to be the characteristic length scale for vortex detachment on a plunging flat plate with different leading edge geometries and a NACA 0012 airfoil. By considering the flow topology, following concepts introduced by Foss (2004), they found that the LEV induced lift on the airfoil drops when fluid begins to recirculate around the trailing edge. An early stage of the flow topology during the LEV growth phase on an unsteady flat plate is schematically depicted in Fig. 1. Here, the LEV is denoted as node $N_{1}$. Recirculation is initiated when the rear stagnation point behind the LEV on the airfoil surface, marked by a green diamond in Fig. 1, travels beyond the trailing edge. The recirculated fluid is entrained between the vortex and airfoil, and finally feeds secondary vortices

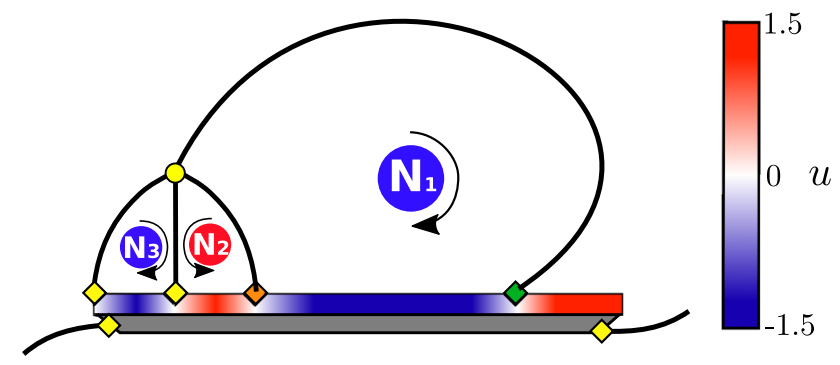

Fig. 1 Sketch of the flow topology during leading edge vortex growth on an unsteady flat plate. Half-saddles are marked with diamonds and full saddle with a circle. The main LEV (node $N_{1}$ ) and secondary vortices (nodes $N_{2}$ and $N_{3}$ ) are highlighted in addition to the tangential velocity on the airfoil surface $u$ induced by them Adapted from Rival et al. (2014)

(nodes $N_{2}$ and $N_{3}$ ) ahead of the main vortex, which grow and interrupt the LEV from its feeding shear layer.

Nevertheless, Sattari et al. (2012) found in a generic experiment that a two-dimensional starting vortex produced by a DBD-PA on the rear edge of a plate detaches from its feeding shear layer in absence of any geometric length scale. This suggests that vortex detachment can occur independent of any length scale. Likewise, Widmann and Tropea (2015) found that for a pitching and plunging flat plate at intermediate $\mathrm{Re}$ and a higher $\mathrm{k}$, in combination with a large effective angle of attack amplitude $\hat{\alpha}_{\text {eff }}$, the LEV stops accumulating circulation before the rear stagnation point behind the vortex reaches the trailing edge. They conclude that $c$ is not the defining length scale for the investigated experimental parameters. Instead a viscous response of the boundary layer between the LEV and the airfoil is identified to cause an abrupt eruption of surface fluid that initiates the growth of secondary vortices ahead of the main vortex. In this detachment scenario these secondary vortices, also referred to as secondary structures, grow and cut off the LEV from its feeding shear layer. From a topological point of view, growing secondary structures (nodes $N_{2}$ and $N_{3}$ in Fig. 1) cause their rear confining stagnation point, marked as an orange diamond in Fig. 1, to travel downstream. They term this locally initiated detachment mechanism 'boundary-layer eruption', which adopts the terminology used by Doligalski et al. (1994). This kind of detachment without recirculation of fluid around the trailing edge, where $c$ is not the characteristic length scale, was also observed by Eslam Panah et al. (2015) and Akkala and Buchholz (2017) for an LEV on a plunging flat plate at high $k$ between 1 and 2 .

A major objective of the present study is to establish a basis for future flow control attempts on LEVs using two different flow actuation mechanisms working with different media. Therefore, experiments were carried out both in water and air under geometric, kinematic and dynamic similarity. The choice of different working media is not only 
related to flow manipulation devices used, but also allows an extended dimensionless parameter range. At first, a common baseline case is defined to enable comparability of the flow field and vortex characteristics between results from the two facilities. Second, with the intention to better understand the formation of secondary structures during LEV growth as well as their consequences regarding the LEV detachment process, the topology of the flow field for different dimensionless parameters and effective angle of attack amplitudes is investigated. Additionally, the emergence of secondary structures is modelled to allow for a precise timing of flow control approaches targeting secondary structure manipulation.

\section{Facilities and methods}

\subsection{Parameter space and facilities}

Experimental investigations of the LEV formation and detachment on a one-shot pitching and plunging flat plate were conducted at two different facilities: a wind tunnel at the Technische Universität Darmstadt (TUDA) and a water tunnel at Beihang University (BUAA). Both set-ups use time-resolved particle image velocimetry (PIV) to characterize the flow field.

At TUDA an open return wind tunnel with a test section of $0.45 \mathrm{~m} \times 0.45 \mathrm{~m}$ was used, whereby the turbulence level measured by hot-wire anemometry was found to be less than $2 \%$ for $U_{\infty}=3.45 \pm 0.05 \mathrm{~m} / \mathrm{s}$. At BUAA a water tunnel with a test section of $1 \mathrm{~m} \times 1.2 \mathrm{~m}$ in spanwise and vertical orientation was used in which the RMS turbulence level was found to be less than $1.3 \%$ for $U_{\infty}=0.2 \mathrm{~m} / \mathrm{s}$. The investigated airfoil at both facilities was a flat plate of less than $6 \%$ thickness and $c=120 \mathrm{~mm}$ with a sharp leading edge of $30^{\circ}$ in order to produce a defined separation of the leading edge shear layer.

The definition of a common baseline case, which enables comparability of results from both facilities, requires a priori consideration of the overlap of parameter spaces. Limiting factors for the maximum reduced frequency and Strouhal number are the maximum available actuator force to move the airfoil and maximum allowable plunge height, considering the airfoil mass and chord length. The Re range is determined by the lowest and highest free-stream velocity attainable in the respective tunnels, in relation to the chord length, while $U_{\infty}$ also influences the $k$ and St ranges. Figure 2 illustrates the attainable parameter ranges in the respective facilities and their overlap for St $=0.1$ and $\hat{\alpha}_{\text {eff }}=30^{\circ}$ with $c=120 \mathrm{~mm}$. Here, the attainable parameter ranges are compared for different $\mathrm{k}$ and Re. St and the effective angle of attack amplitude $\hat{\alpha}_{\text {eff }}$ were additionally considered for the definition of the common baseline case.

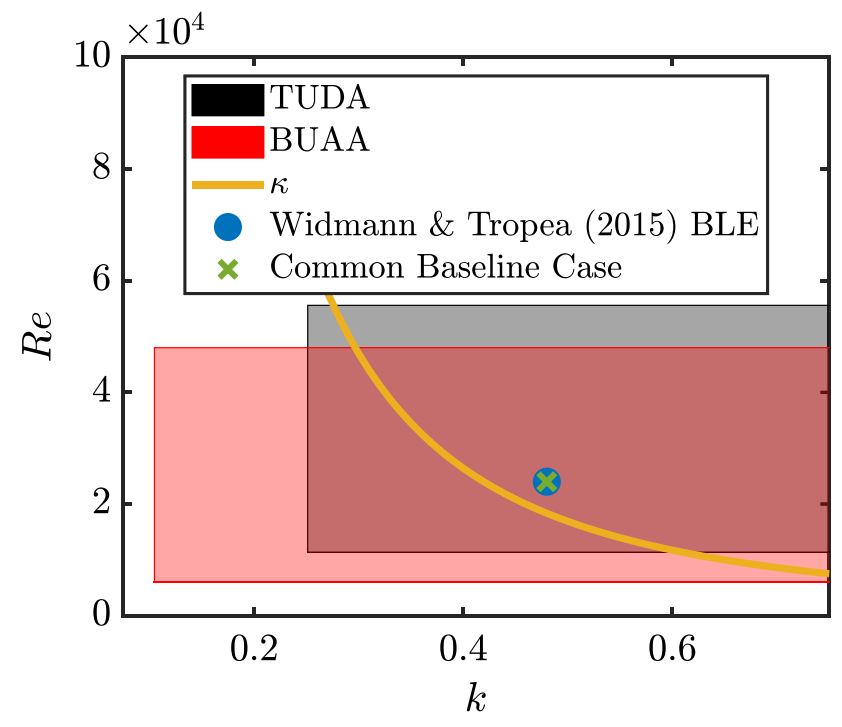

Fig. 2 Dimensionless parameter ranges of facilities at BUAA and TUDA for $\mathrm{St}=0.1, \hat{\alpha}_{\mathrm{eff}}=30^{\circ}$ and $c=120 \mathrm{~mm}$. The covering ratio $\kappa$ introduced by Widmann and Tropea (2015), differentiating between detachment mechanisms, is indicated with a curve in addition to their condition where boundary-layer eruption detachment (BLE) was observed. Finally, the chosen common baseline case for this study is shown

The dimensionless and geometric parameters of the common baseline case have been chosen such that a boundarylayer eruption detachment mechanism of the LEV can be expected, as discussed in Sect. 1. Widmann and Tropea (2015) developed an analytical parameter, which enables the identification of dimensionless parameters that lead to boundary-layer eruption detachment, termed the dimensionless covering ratio $\kappa$. This parameter is based on the assumption that if the LEV is large enough to cover the entire airfoil chord, recirculation around the trailing edge is initiated. The coverage of the LEV is determined by an approximation of the mass flux into the LEV, which is in turn dependent on the shear layer thickness. The shear layer thickness is approximated using the Falkner-Skan solution, which takes the leading edge curvature into account. Through a series of parameter variations they find a threshold value of $\kappa$ at which transition between detachment mechanisms occurs. This threshold is illustrated in Fig. 2 as an orange line. For cases with parameters located above this line, the LEV should detach due to boundary-layer eruption. One case where a boundary-layer eruption was identified at $k=$ $0.48, \operatorname{Re}=24,000, \mathrm{St}=0.1$ and $\hat{\alpha}_{\text {eff }}=30^{\circ}$ for $c=120 \mathrm{~mm}$ is depicted with a blue dot. This is chosen as the common baseline case for both facilities in the current study.

An additional parameter that has not been considered in existing LEV detachment models and is not taken into account for in $\kappa$ is the history of the effective angle of attack $\alpha_{\text {eff }}(t)$. It affects the shear layer and LEV characteristics most 
directly through the vertical velocity component at the leading edge. The magnitude of $\alpha_{\text {eff }}(t), \hat{\alpha}_{\text {eff }}$, is determined by the addition of the inflow angle induced by the plunging motion $\alpha_{\text {plunge }}(t)=\dot{h}(t) / U_{\infty}$ and the geometric angle of the airfoil due to the pitching motion $\alpha_{\text {geo }}(t)$, as shown in Fig. 3 for the common baseline case with $\hat{\alpha}_{\text {eff }}=30^{\circ}$. By adapting $\alpha_{\text {geo }}(t)$ for different dimensionless parameter values, $\hat{\alpha}_{\text {eff }}$ can be kept constant. Additionally, different $\hat{\alpha}_{\text {eff }}$ can be produced independent of the dimensionless parameters. All kinematics investigated in this study are designed with a quasi-sinusoidal evolution of $\alpha_{\text {eff }}(t)$. As an extract from cyclic motion, only the downstroke of the airfoil is investigated. The phase offset between the pitching and plunging motion was set to zero to allow results to be compared with previous studies of the LEV detachment.

The execution of combined pitching and plunging kinematics at TUDA was realized by attaching the flat plate to two linear actuators of type LinMot PS01-48x240F-C with the aid of a midspan bracket, one at the leading edge and a second one at about $x / c=0.68$. By execution of different motion profiles on both actuators, pitching motion could be added to pure plunge motion. To produce an accurate motion profile and vibration free translation of the airfoil, the position and acceleration of the actuators were used by the actuator control as a feedback loop input.

A customized experimental platform, including a rotating stage and a linear translation stage, was designed to enable the combined pitching and plunging motion of the flat plate at BUAA. The linear translation of the investigated airfoil to produce plunging motion was realized with a servo motor of

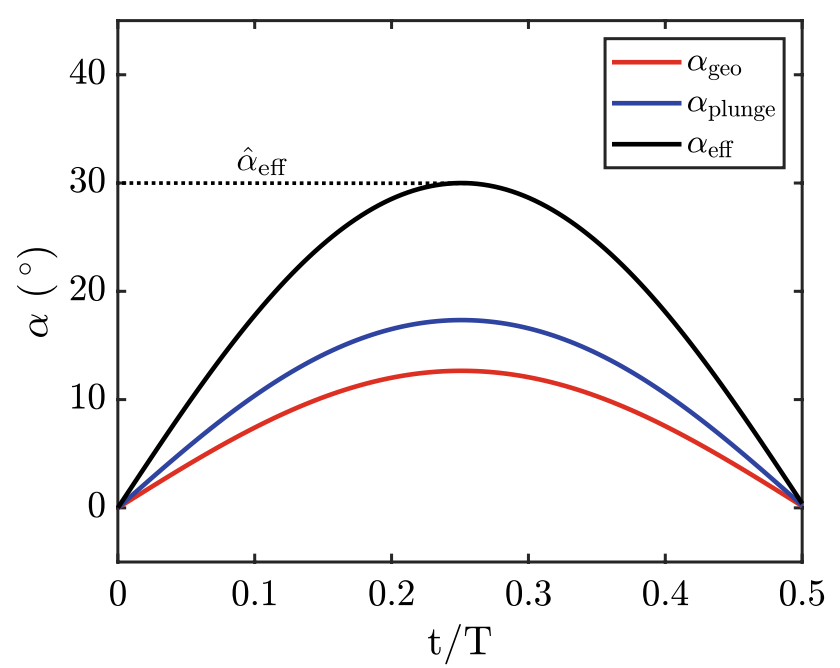

Fig. 3 Evolution of the effective angle of attack $\alpha_{\text {eff }}(t)$ during the downstroke as a result of the superposition of the induced angle of attack due to the plunging motion $\alpha_{\text {plunge }}(t)$ and the geometric angle of attack $\alpha_{\text {geo }}(t)$ as a function of dimensionless time $(t / T)$ for the common baseline case. The effective angle of attack amplitude $\hat{\alpha}_{\text {eff }}$ is additionally indicated type YASKAWA SGM7J-100 and a ball screw rod (LC-EA030A). Rotational motion of the airfoil was realized with a servo motor of type YASKAWA SGM7J-400 and a decelerator (KAMO JFR90), which are both directly connected to the airfoil. A programmable multi-axis controller (Delta Tau Clipper) was used to synchronize all the servomotors.

To allow flow fields to be compared between both facilities, the vertical leading edge position of the airfoil $h(t)$ was extracted from masked raw PIV images via image processing. In Fig. 4, $h(t)$ of both facilities and the intended curve are shown for the common baseline case, normalized with the respective full stroke height $H$.

Deviations between the measured and intended leading edge positions were found to be smaller than $0.6 \%$ of the full stroke height for all investigated cases, which is within the accuracy of the actuators used to pitch and plunge the flat plates. These deviations are small enough to allow velocity fields and vortex characteristics from both setups to be directly compared to one another.

After establishing good agreement between results from both facilities for the baseline case, a larger parameter range was tested at both facilities to characterise secondary structure emergence and their dynamics during vortex detachment. Although the dimensionless parameter range covered by this study is within the range of MAVs, it is also assumed to be transferable to biological propulsion as discussed and quantified in Sect. 1. The Reynolds number was fixed at 24,000 for all experiments while the reduced frequency was varied between 0.3 and 0.48 and the Strouhal number between 0.04 and 0.16 . Table 1 lists all cases investigated at TUDA including the common baseline case (ID 3 ) with their dimensionless parameters and the geometric parameters $\hat{\alpha}_{\text {eff }}$ and $\hat{\alpha}_{\text {geo }}$. Additionally, the motion period of the entire cycle

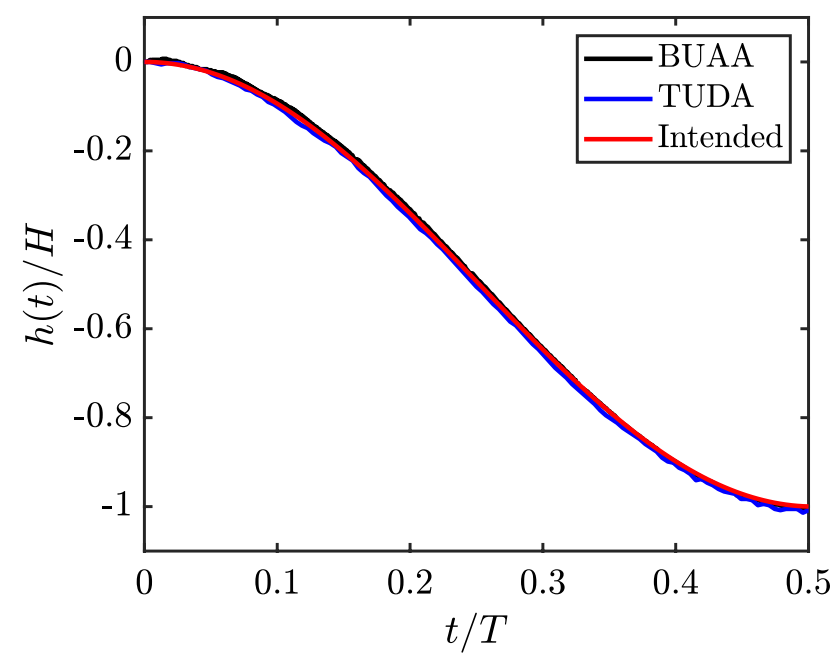

Fig. 4 Comparison of the intended and experimentally realised vertical leading edge positions $h$, normalized by the plunge height $H$ as a function of dimensionless time $(t / T)$ for the common baseline case 
Table 1 Dimensionless and geometric parameter range investigated at TUDA for $\mathrm{Re}=24,000$, including the common baseline case (ID 3)

\begin{tabular}{|c|c|c|c|c|c|c|c|}
\hline ID & Sym. & $k$ & $\begin{array}{c}\mathrm{T} \\
(\mathrm{ms})\end{array}$ & $S t$ & $\begin{array}{c}H \\
(\mathrm{~m})\end{array}$ & $\begin{array}{c}\hat{\alpha}_{\text {eff }} \\
\left({ }^{\circ}\right)\end{array}$ & $\begin{array}{c}\hat{\alpha}_{\text {geo }} \\
\left({ }^{\circ}\right)\end{array}$ \\
\hline \hline 1 & $\bullet$ & 0.48 & 236 & 0.04 & 0.016 & 30 & 22.69 \\
\hline 2 & & 0.48 & 236 & 0.08 & 0.031 & 30 & 16.07 \\
\hline 3 & & 0.48 & 236 & 0.1 & 0.039 & 30 & 12.67 \\
\hline 4 & $\circ$ & 0.48 & 236 & 0.1 & 0.039 & 20 & 2.67 \\
\hline 5 & 0 & 0.48 & 236 & 0.08 & 0.031 & 20 & 6.07 \\
\hline 6 & 0 & 0.48 & 236 & 0.04 & 0.016 & 20 & 12.7 \\
\hline 7 & $\square$ & 0.3 & 377 & 0.08 & 0.05 & 30 & 15.95 \\
\hline 8 & $\square$ & 0.3 & 377 & 0.04 & 0.025 & 30 & 22.86 \\
\hline 9 & $\square$ & 0.3 & 377 & 0.08 & 0.05 & 20 & 5.95 \\
\hline 10 & $\square$ & 0.3 & 377 & 0.04 & 0.025 & 20 & 12.86 \\
\hline
\end{tabular}

Table 2 Dimensionless and geometric parameter range investigated at BUAA for $\operatorname{Re}=24,000$, including the common baseline case (ID 11)

\begin{tabular}{|c|c|c|c|c|c|c|c|}
\hline ID & Sym. & $k$ & $\begin{array}{c}\mathrm{T} \\
(\mathrm{ms})\end{array}$ & $S t$ & $\begin{array}{c}H \\
(\mathrm{~m})\end{array}$ & $\begin{array}{c}\hat{\alpha}_{\text {eff }} \\
\left({ }^{\circ}\right)\end{array}$ & $\begin{array}{c}\hat{\alpha}_{\text {geo }} \\
\left({ }^{\circ}\right)\end{array}$ \\
\hline \hline 11 & $\searrow$ & 0.48 & 3927 & 0.1 & 0.039 & 30 & 12.67 \\
\hline 12 & $*$ & 0.48 & 3927 & 0.16 & 0.063 & 30 & 3.31 \\
\hline 13 & $\triangle$ & 0.3 & 6285 & 0.04 & 0.025 & 20 & 12.86 \\
\hline
\end{tabular}

$T$ and the full plunge height $H$ are listed. The ID assigned to each case will be used for identification in subsequent evaluations. The color, shape and fill of markers is chosen to allow for a differentiation of parameter variations: The color represents the Strouhal number and is coded from blue to red to yellow to green for increasing Strouhal numbers. The shape of markers marks the reduced frequencies, where round symbols represent $k=0.48$ and rectangles $k=0.3$. Cases with $\hat{\alpha}_{\text {eff }}=30^{\circ}$ can be identified by filled markers and cases with $20^{\circ}$ by open symbols.

The St-range at TUDA was limited to below 0.1 due to high inertial forces of fast motion kinematics in air. The difference in dynamic motions between facilities becomes evident by considering the motion period $T$ for the common baseline case listed in Tables 1 and 2, which differ by an order of magnitude. By including a set of cases with $\hat{\alpha}_{\text {eff }}=20^{\circ}$ (ID 4-6 and 9-10) and variations of St (ID 1-3) and $\mathrm{k}$ (ID 7 and 8 ) with respect to the baseline case, effective inflow angles close to pure plunging motion, indicated by their low $\hat{\alpha}_{\text {geo }}$, can also be investigated. The parameter space investigated at BUAA is depicted in Table 2, again including the common baseline case referred to ID3 (TUDA) and ID 11 (BUAA). With the higher attainable Strouhal number the dimensionless parameter range at BUAA could be extended towards the regime of efficient forward flight, characterized by higher St. It is notable that ID 10 and 13 match in all key parameters even though they will not be examined in detail below.

At TUDA raw PIV images were acquired with a double frame recording frequency of $1 \mathrm{kHz}$ and an inter-framing time of $\triangle t=150 \mu \mathrm{s}$, yielding a particle displacement between 2 and 8 pixels for PIV correlations. DEHS seeding particles with a mean diameter of $0.5-1 \mu \mathrm{m}$ were introduced into the settling chamber of the wind tunnel. Their response time $\tau_{s}$ was computed to be $2.7 \mu$ s according to Raffel et al. (2007). Compared to the minimum time scale of the flow, estimated by the convective time of the Kolmogorov length scale $\eta_{K}=c \times \mathrm{Re}^{-3 / 4} \approx 60 \mu \mathrm{m}$ with $c$ as the macro-scale reference, $\tau_{F}=\eta_{K} / U_{\infty}=20 \mu$ s, the response time is considered to be adequate. A Photron SA1.1 High Speed Camera and a Carl Zeiss lens of $50 \mathrm{~mm}$ focal distance with an aperture of $f=2.2$ captured images of the seeded flow around the flat plate with a resolution of $1024 \times 1024$ pixels. The field of view spanned by a light sheet plane of $3 \mathrm{~mm}$ thickness, located at $28 \%$ span from the wind tunnel wall, was $1.9 c$ $\times 1.9 c$, resulting in a spatial resolution of $4.556 \mathrm{px} / \mathrm{mm}$. The seeded flow field was illuminated using a dual cavity Litron DY-303 Nd:YLF laser with a single pulse energy of $18 \mathrm{~mJ}$. Image correlations were performed with PIVview 2C software from PIVTEC GmbH utilizing a multi-grid, multipass interrogation scheme including a sub-pixel routine at an initial interrogation area (IA) size of $64 \mathrm{px} \times 64 \mathrm{px}$ and a final of $12 \mathrm{px} \times 12 \mathrm{px}$ at $50 \%$ overlap, yielding 92 IA's over the chord. Outliers were identified by a median test (cf. Westerweel and Scarano 2005) in a $3 \times 3$ neighbourhood with a threshold of twice the velocity magnitude and found to be always less than $1.6 \%$ of all computed vectors for all acquired image pairs. Each parameter set was recorded ten times, yielding a spatially averaged standard deviation of flow fields within $3.1 \%$ and a temporally averaged deviation of vortex characteristics of $2.1 \%$ of the respective asymptotic values. Standard deviation values were computed by using bootstrap testing with 1000 bootstrap samples for each investigated number of repetitions (cf. Benedict and Gould 1996).

In the BUAA setup a high-speed CMOS camera of type Photron Fastcam SA2/86K-M3 fitted with a Nikon lens of $50 \mathrm{~mm}$ focal length was used to acquire time-resolved PIV raw images. Image pairs were acquired at a frequency of $200 \mathrm{~Hz}$ with a resolution of $2048 \mathrm{px} \times 2048 \mathrm{px}$. The seeded flow field was illuminated by a continuous Nd-YAG laser with $8 \mathrm{~W}$ nominal power at midspan position with a laser light sheet of $3 \mathrm{~mm}$ thickness. Hollow glass beads with a median diameter of $20 \mu \mathrm{m}$ and a density of $1.05 \mathrm{~g} / \mathrm{cm}^{3}$ were used as seeding particles in water. For final evaluations, the same correlation algorithm from TUDA was used, where the final IA size was $16 \mathrm{px} \times 16 \mathrm{px}$ with $50 \%$ overlap resulting in about 82 velocity data points over the airfoil chord.

\subsection{Data processing}

The circulation and position of the LEV are characteristic parameters that can be compared between both setups and 
used for further evaluations. To investigate influences of the evaluation method used to obtain circulation and position, different methods have been tested on the same set of raw images. Investigated methodologies to obtain LEV circulation are based on the identification of regions belonging to the vortex prior to a first order spatial integration of vorticity according to Stokes's theorem. Vortex characteristics were extracted from single runs before they were ensemble averaged.

LEV boundaries computed using the $\lambda_{c i}$ method by Zhou et al. (1999) were found to be strongly dependent on the threshold used to identify the vortex, whereas the Q criterion by Hunt et al. (1988) identified only an inner vortex core while excluding outer vortex regions. Additionally, both methods were found to intermittently attribute the leading edge shear layer to the main vortex, leading to strong fluctuations of the subsequently determined circulation. The LEV boundary computed by thresholding the $\Gamma_{2}$ scalar field, introduced by Graftieaux et al. (2001), which considers regions of pure shear as the vortex area, was found to identify the vortex boundary most consistently for both data-sets using the default threshold of $\Gamma_{2}=2 / \pi$. Therefore, it was used to quantify the circulation evolution of the LEV from velocity fields obtained at both facilities. This was done by integrating vorticity within the detected LEV boundary according to Stokes's theorem. The detection of the LEV center from maxima of the $\Gamma_{1}$ scalar function, also introduced by Graftieaux et al. (2001), as well as from the Q criterion, were found to be reliable, at least with respect to visual inspection of instantaneous vector fields. Although the $\Gamma_{1}$ function is not Galilean invariant by definition, deviations from the Galilean invariant Q criterion were found to be less than $2 \%$, which in turn provides evidence that the chosen plate-fixed frame of reference allows reasonable interpretation of the extracted topology.

Even when using the same vortex boundary identification and circulation computation method, as well as spatial vorticity derivation schemes, the LEV circulation evaluation was found to be dependent on the cross-correlation parameters used to obtain velocity data from the same raw images in PIV correlations. The LEV circulation obtained with identical post-processing routines but different correlation algorithms, a gradient-based cross-correlation optimization based on the Lucas-Kanade method (cf. Champagnat et al. 2011) and a standard FFT correlation algorithm, differs up to $9 \%$. The maximum circulation divergence occurs when the LEV starts to decay, which is accompanied by a blurred outer boundary leading to different areas identified as a vortex. This highlights that PIV correlation schemes influence the circulation computation within the vortex domain significantly, even when using the same vortex identification method for boundary detection, vorticity derivation and subsequent spatial integration procedures. When aiming to compare vortex characteristics between different facilities, these deviations can hinder comparability of characteristics obtained from flow fields. Consequently all evaluations have been performed using the same correlation software and parameters according to the TUDA setup described in Sect. 2.1. Data sets in terms of correlated velocity fields of the baseline cases from TUDA and BUAA (ID3 and ID 11) are available online as reference cases (http://dx.doi. org/10.25534/tudatalib-168).

With Eulerian vortex identification methods the LEV detachment, related to changes of the flow topology, can only be investigated implicitly by observation of effects on vortex characteristics. In contrast, Lagrangian methods in terms of coherent structures allow direct identification of topological changes of the flow field initiating vortex detachment. In this study coherent structures are identified with the aid of finite-time Lyapunov exponent (FTLE) ridges, following the concept and methods introduced by Haller (2002) and Shadden et al. (2006). Ridges of repelling and attracting fluid regions are obtained by thresholding forward-time and backward-time FTLE scalar fields. Topological changes of the flow field can be identified directly by tracking Lagrangian saddle points of the flow field, which are intersection points of forward-time and backward-time FTLE ridges, as shown by Huang and Green (2015). In this study the FTLE computation package developed by Peng and Dabiri (2009) is used to obtain FTLE fields.

In advance of FTLE evaluations, the impact of different parameters governing the results of FTLE computations, including spatial and temporal resolution of the velocity information as well as the integration time $\tau$ were investigated. Computation of physically relevant FTLE ridges requires a sufficient spatial and temporal resolution of the velocity information used for calculations. Both resolutions were tested for current FTLE computations by a reduction of the temporal and spatial resolution by a factor of 2 . In both cases no significant changes were observed in FTLE fields, so both resolutions are assumed to be sufficient. FTLE scalar fields are obtained over a time frame, which is referred to as the integration time $\tau$. For further processing, raw FTLE scalar fields are thresholded using a percentage of maximum FTLE values in each frame to evaluate distinct ridges $(80 \%$ in this study). As mentioned by Peng and Dabiri (2009) $\tau$ does not have an impact on the FTLE ridge topology in terms of their location, but on the strength of ridges via their resolution. Note however that the above statement only holds beyond a minimum $\tau$ that allows sufficient temporal development of LCS instead of quasi-streamline-based LCS determination of separatrices for $\tau \rightarrow 0$ (see e.g. Perry and Chong 1987). The efforts of the present FTLE calculation center around the formation of secondary structures, which have a lifetime of approximately $0.25 \mathrm{~T}$. A final integration time of $\tau=0.11 T$ was chosen accordingly, where the extracted LCS 
comprise all significant features of raw secondary structure contours at an acceptable computational effort. Due to the robustness of FTLE calculations against interpolation errors (cf. Haller 2002), velocity vector fields can be interpolated to obtain highly resolved FTLE fields. In this study the spatial interpolation is chosen based on the trade-off between resolution and computational costs. In final evaluations the spatial resolution of FTLE fields was twice as high as the resolution of the velocity vector fields, since ridges were clearly represented and computational costs were acceptable.

\section{Results}

With the aim of comparing results from the two different facilities at BUAA and TUDA in water and air, this section focuses on the evaluation of the common baseline case.

\subsection{Flow fields}

A qualitative aspect of comparability is the evolution of the flow field and its topology, which is depicted in terms of ensemble averaged vorticity fields in Fig. 5. The coordinate system is fixed at the initial top position of the airfoil leading edge and both axes are normalized by the flat plate chord. At $t / T=0.25$ the LEV containing concentrated negative (blue color coded) vorticity grows on the airfoil by accumulating circulation from the leading edge shear-layer (Fig. 5a, e). A thin layer of counterclockwise rotational fluid, indicated by the positively signed (red color coded) vorticity, can be observed below the vortex in addition to a distinct region comprising positively signed vorticity ahead of the main vortex. This counterclockwise rotational fluid forms a secondary vortex rotating in the opposite direction of the clockwise rotating LEV. A third clockwise rotating vortex between the leading edge shear layer and the secondary vortex completes the secondary vortex structures. This is identifiable from the indicated velocity vectors in Fig. 5d and $\mathrm{h}$ later in the downstroke. At this early stage the flow fields from both setups are in very good agreement with respect to the observed topology and vorticity intensity within the vortices.

Furthermore, towards the end of the downstroke, the LEV continues growing, although at $t / T=0.35$ the connection between vortex and leading edge shear layer appears to be interrupted in the vorticity fields from BUAA (Fig. 5b),

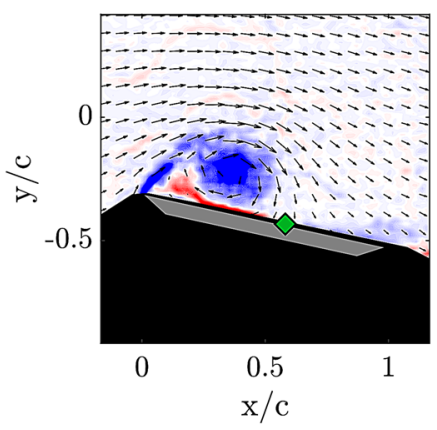

(a) BUAA; $t / T=0.25$

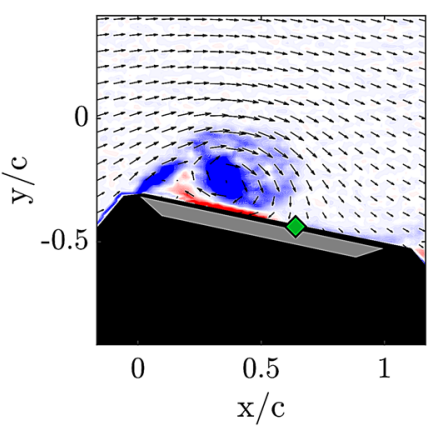

(e) TUDA; $t / T=0.25$

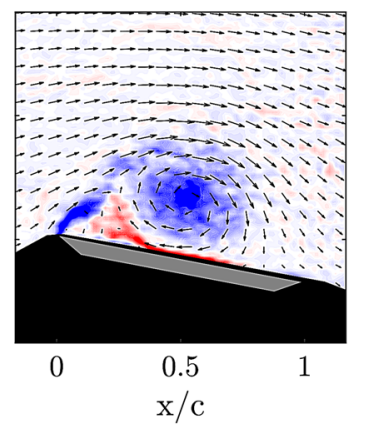

(b) $\mathrm{BUAA} ; t / T=0.35$

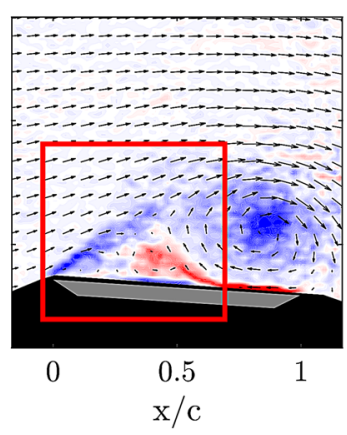

(c) BUAA; $t / T=0.45$

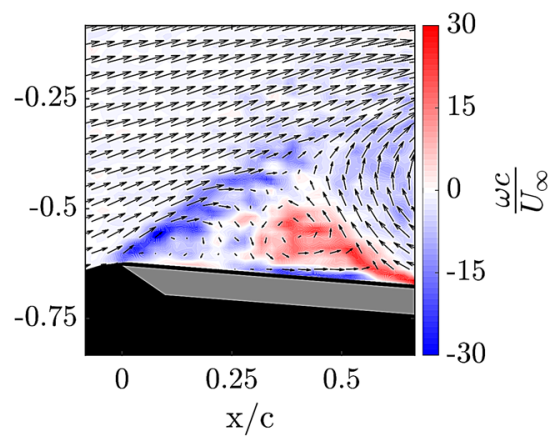

(d) BUAA; $t / T=0.45$

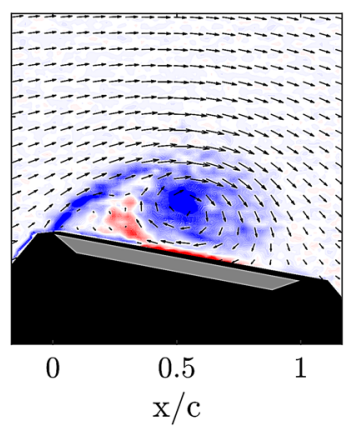

(f) TUDA; $t / T=0.35$

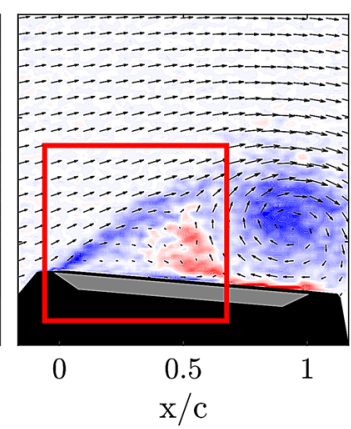

(g) TUDA; $t / T=0.45$

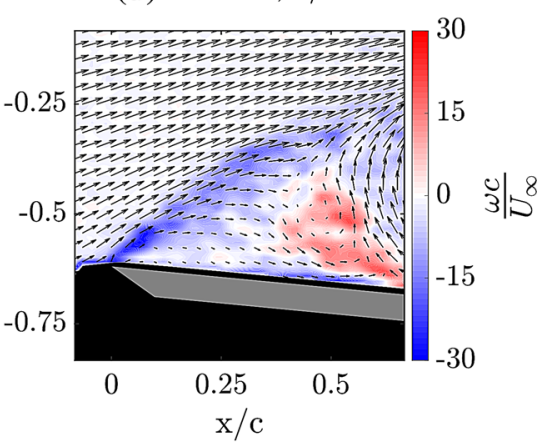

(h) TUDA; $t / T=0.45$
Fig. 5 Flow field in terms of normalized vorticity $\omega c / U_{\infty}$ for different dimensionless time instants $t / T$ from ensemble averaged PIV measurements at BUAA (a-d) and TUDA (e-h) for the common baseline case (ID 11 case from BUAA and ID 3 from TUDA). The inflow is from the left, the airfoil is masked out in grey and the laser light shadow caused by the airfoil in black. While $\mathbf{a}-\mathbf{c}$ and $\mathbf{e}-\mathbf{g}$ depict the entire field with only every 6 th vector for clarity, $\mathbf{d}$ and $\mathbf{h}$ show the leading edge region with every 3rd vector as highlighted in $\mathbf{c}$ and g. In a and e the rear confining stagnation point behind the LEV is marked with a green diamond 
while it is unimpaired in the TUDA fields (Fig. 5f). An inspection of the entire time series of the flow fields revealed that the LEV and shear layer are frequently interrupted for TUDA results, while for BUAA flow fields their connection appears to be generally weaker, indicated by lower vorticity values of the connecting region. Differences of single events in the instantaneous velocity fields are of minor relevance for the present study, aimed at highlighting the main features of the ensemble averaged velocity fields. Shortly before the end of the downstroke at $t / T=0.45$, a slight deviation of the LEV center position can be observed comparing Fig. 5c and g. At this late stage of the downstroke the LEV is only weakly connected to the leading edge shear layer for both evaluations, indicating the end of circulation accumulation. Overall, the topological evolution of the ensemble averaged flow fields and the qualitative vortex characteristics evolution are in very good agreement between the two facilities.

\subsection{Vortex characteristics}

The LEV characteristics in terms of its position, size and circulation determine the lift that is induced on the pitching and plunging flat plate. Therefore, these quantities are of key interest in future flow control approaches. To allow comparability between different flow control approaches at both facilities, vortex characteristics of the common baseline case will now be compared quantitatively. Figure 6 depicts the normalized LEV circulation $\Gamma_{\mathrm{LEV}} / U_{\infty} c$ determined by the integration of vorticity within its detected boundary of the $\Gamma_{2}$ scalar field. Raw images were correlated using the

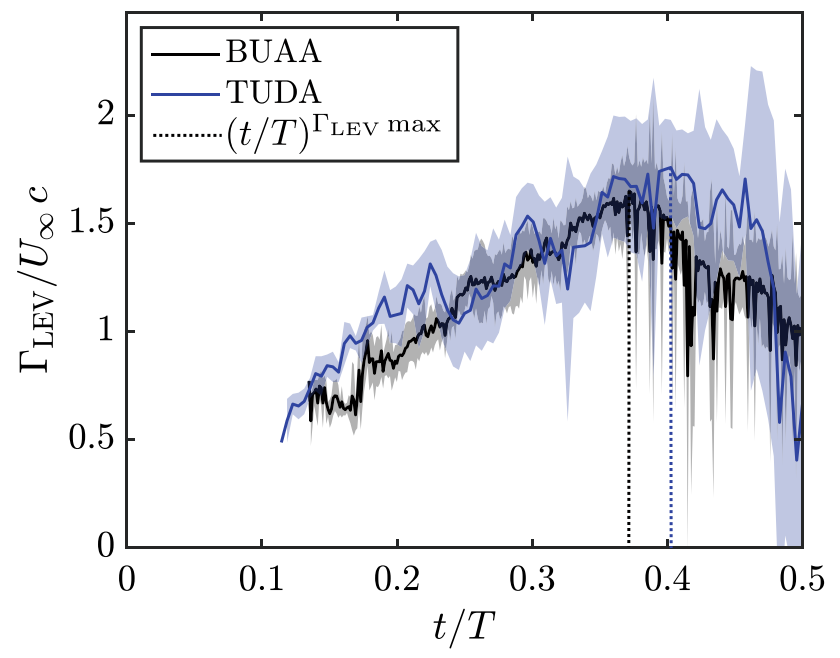

Fig. 6 Evolution of the normalized leading edge vortex circulation $\Gamma_{\mathrm{LEV}} / U_{\infty} c$ for the common baseline case from both setups. The respective standard deviation is indicated as a coloured shadow and the peak circulation $(t / T)^{\Gamma_{\mathrm{LEV}} \max }$ as dashed line. Circulation is obtained by integration of vorticity over the vortex area identified from the $\Gamma_{2}$ scalar field same algorithm implemented at TUDA and the same vortex identification method in addition to the same vorticity derivation and integration schemes. The circulation of the vortex was computed for each individual run prior to ensemble averaging of the extracted circulation for all ten runs. In this manner the standard deviation can be computed for the final, ensemble averaged results, indicated as coloured shading in Fig. 6. Early during vortex growth, the LEV circulation from both facilities increases with the same slope but with a somewhat higher vortex circulation magnitude of the TUDA results before $t / T \approx 0.22$. After this phase, stronger normalized circulation fluctuations are observed in the TUDA results compared to those from BUAA. These fluctuations and the initial offset originate from an intermittent inclusion of the leading edge shear layer into the computational vortex boundary, evident in single frame evaluations, which are only observed for TUDA experiments. This was confirmed by simultaneous fluctuations of the detected area of the vortex boundary. The LEV stops accumulating circulation between $0.38 \leq t / T \leq 0.41$ in BUAA and TUDA results, indicated by the peak circulation instants $(t / T)^{\Gamma_{\mathrm{LEV}} \max }$ (dashed lines in Fig. 6).

To verify that intermittent inclusion of the leading edge shear layer into the calculation of the circulation is an error source for deviations of the circulation evolution during the early LEV growth phase, the circulation was also computed using the entire field of view as an integration domain. A comparison of the normalized circulation from the entire field of view for both setups is shown in Fig. 7. Despite very small deviations of the circulation, the overall quantitative agreement of the evolution is very good. In both

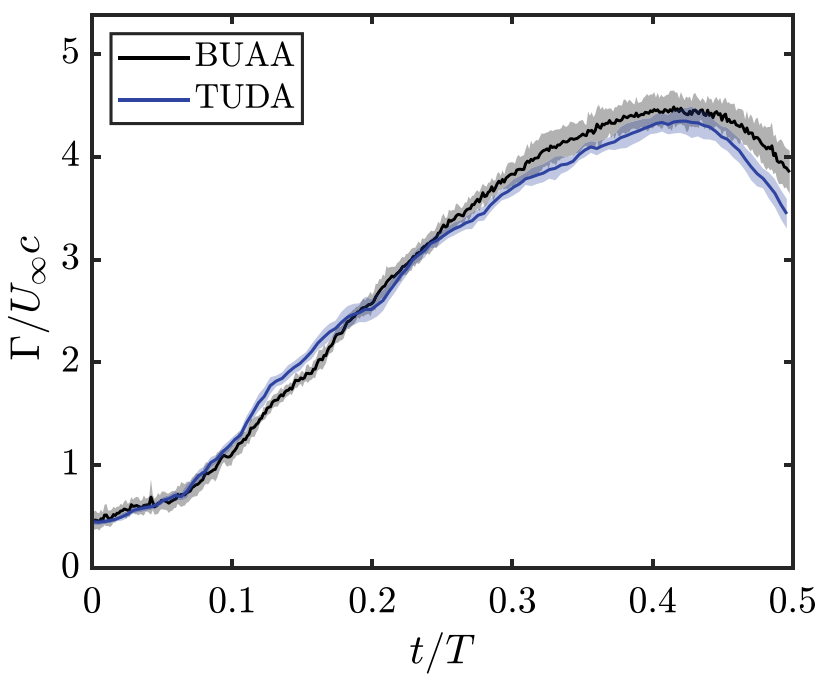

Fig. 7 Evolution of the normalized circulation $\Gamma / U_{\infty} c$ for the common baseline case evaluated from the entire field of view to identify vortex boundary detection as the error source for circulation deviations. The standard deviation is indicated as coloured shadows 
experiments (at BUAA and TUDA), the maximum circulation is reached between $0.4151 \leq t / T \leq 0.4195$ with $3 \%$ amplitude difference. Deviations in circulation remain below $5.3 \%$ at any instant throughout the downstroke. Based on this good quantitative agreement it can be concluded that the observed deviations of circulation evolution in Fig. 6 are caused by an intermittent inclusion of the leading edge shear layer into the integration domain for TUDA results during vortex identification. Potential reasons for these deviations could be the different spatial resolution of velocity fields or different free-stream turbulence levels of the tunnels used.

Figure 8 shows a comparison of the normalized streamwise LEV center position in a plate-fixed frame of reference. Again, the position is extracted from phase-locked velocity fields taken at each individual run prior to ensemble averaging. The evolution is in good agreement up to $t / T=0.39$, whereupon small deviations of the position between BUAA and TUDA results occur. Since the accumulation of circulation in the vortex was found to stop at $t / T \approx 0.4$, these deviations are attributed to the final convection of the vortex downstream of the airfoil and thus are not of interest for the current study. Note however, that the vortex center evolution in TUDA results might be considered as more parabolic.

Overall, good agreement of vortex characteristics is achieved between ensemble averaged results derived from the experiments performed at BUAA and TUDA.

\subsection{Detachment mechanism}

Two different mechanisms of LEV detachment are postulated in literature, as pointed out in Sect. 1. One is related to fluid recirculation around the trailing edge of the airfoil,

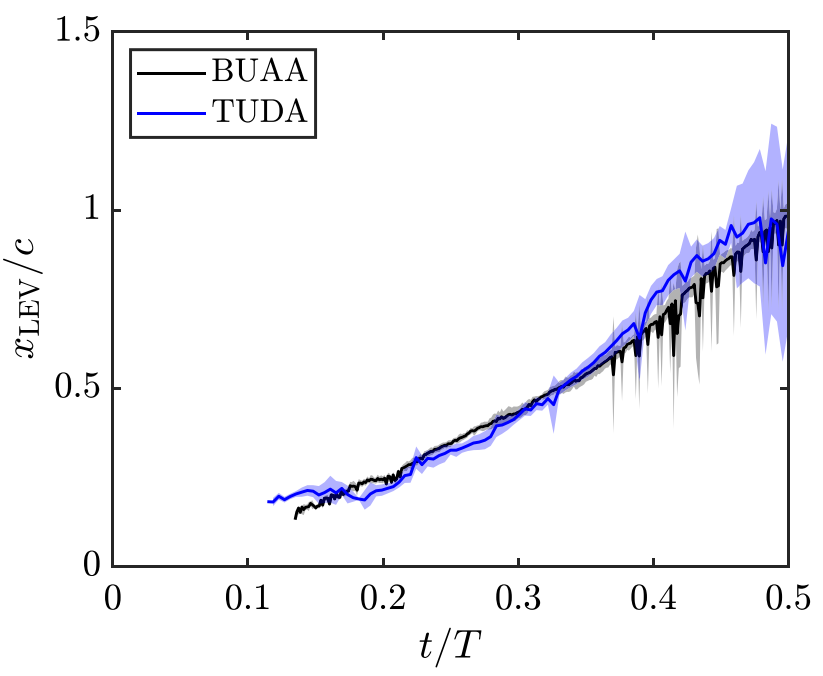

Fig. 8 Evolution of the normalized streamwise LEV center position $x_{\mathrm{LEV}} / c$ in a plate-fixed frame of reference from the maximum of the $\Gamma_{1}$ scalar field for the common baseline case. The standard deviation is indicated as a coloured shadow when the rear stagnation point of the flow behind the LEV on the airfoil convects beyond the trailing edge. This mechanism is investigated in the common baseline case of this study. As demonstrated by Rival et al. (2014), the extraction of the velocity immediately above and parallel to the airfoil surface allows the convection of the rear confining stagnation point of the LEV (marked as green diamond in Figs. 1 and 5a, e) to be tracked according to the change of velocity sign. The tangential velocity induced by vortices on the airfoil surface is schematically shown in Fig. 1 for an arbitrary point during airfoil motion. By identification of the rear stagnation point evolution, the temporal instant of maximum circulation can be compared to that of recirculation.

Figure 9 shows tangential velocity distributions on the airfoil surface over dimensionless time. Velocities are extracted along three lines parallel to the surface and running tangential to the plate from the leading edge at $x / c=$ 0 to the trailing edge at $x / c=1$ for time steps of $\Delta(t / T) \approx$ 0.002 . Figure 9 shows averaged values of these three lines that are located $y / c=0.011$ apart from each other with $y / c=0.011$ spacing to the surface.

Red colour coded areas indicate downstream velocities while blue colour coded areas velocity represents upstream fluid motion induced by the clockwise rotating LEV on the airfoil surface. So a LEV driven upstream velocity region can be clearly identified in Fig. 9 convecting downstream on the airfoil. Upstream velocities on the airfoil surface occur from about $t / T \approx 0.1$ for both evaluations. After emergence of the LEV a change of sign in velocity can be observed downstream of the vortex, which indicates the rear stagnation point of the flow at the rear of the LEV. For clarity it is marked with a green dashed line in Fig. 9. During the growth of the LEV, the stagnation point travels downstream until it reaches the trailing edge at $x / c=1$ (instant marked with a black square). From this instant on, recirculation of fluid from beneath the airfoil around the trailing edge is initiated. This instant agrees well between the two facilities: $t / T=0.39$ for BUAA and $t / T=0.38$ for TUDA. Reconsidering the instant at which circulation accumulation stops, identified from the LEV circulation evolution (at $t / T \approx 0.39$ ), the recirculation of fluid occurs slightly before peak circulation. This shows that for the investigated baseline case, the termination of circulation accumulation of the LEV correlates with recirculation of fluid around the trailing edge for both facilities. The correlation indicates that the LEV detaches in accordance with the process described by Rival et al. (2014) in the baseline case. Considering the covering ratio $\kappa$, a detachment in accordance with the boundary layer eruption detachment was expected. A potential reason for the encountered deviation of the detachment mechanism could be the leading edge curvature of the airfoil, which affects the shear layer properties 


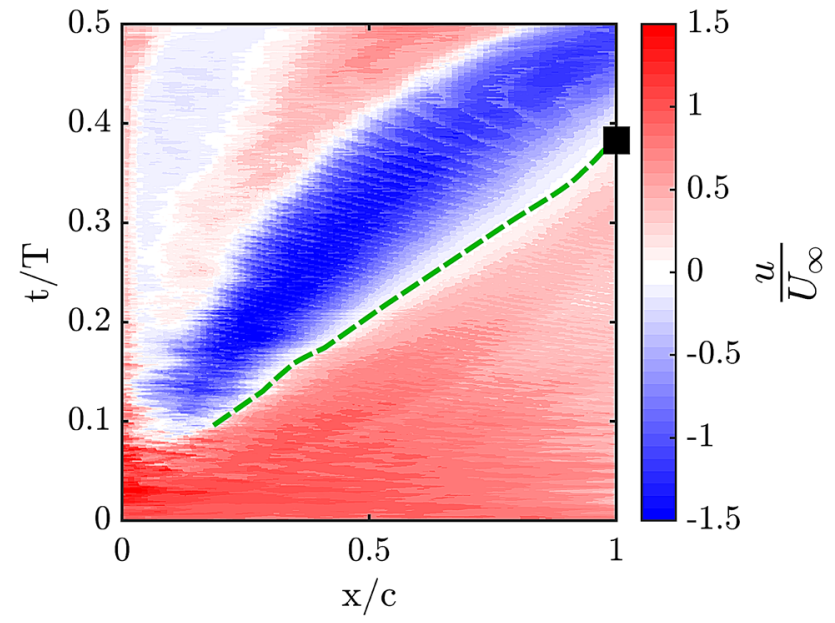

(a) BUAA

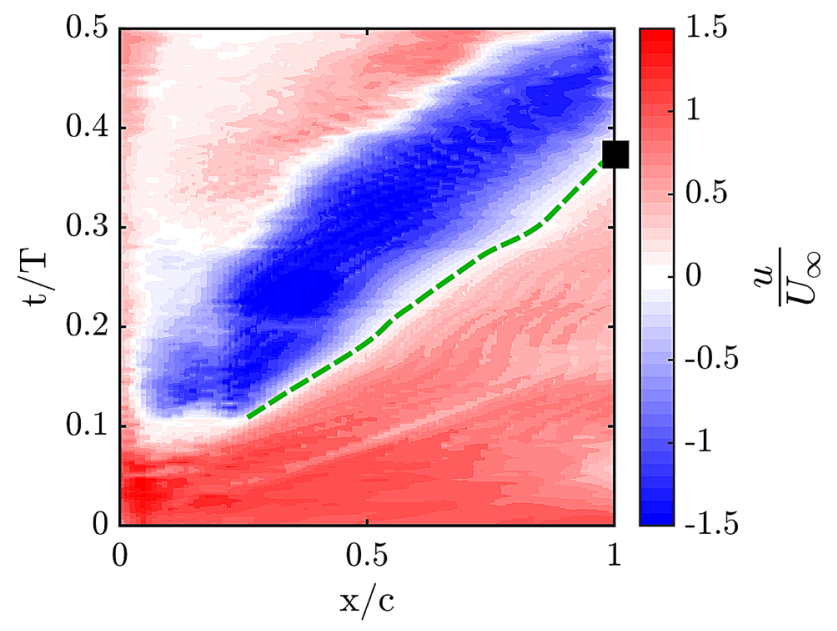

(b) TUDA

Fig. 9 Evolution of the velocity tangential to the airfoil surface $u$ scaled by the free-stream velocity $U_{\infty}$ over dimensionless chordwise position and time. The trace of the rear stagnation point of the flow behind the LEV is marked with a green dashed line; a tangential velocity from measurements in water at BUAA; $\mathbf{b}$ tangential velocity from measurements in air at TUDA

and thus the circulation accumulation rate of the LEV. Nevertheless, it should be noted that distinct secondary structures upstream of the LEV can be observed at later instants for $t / T \geq 0.2$, indicated by additional changes of tangential velocity sign in horizontal direction in Fig. 9.

Since ID 10 and ID 13 cases are also at the same dimensionless parameters, they were also compared using all measures from the baseline case examination. In accordance with the comparisons from above, the agreement of ID 10 and 13 cases is similarly good.

\section{Discussion}

The occurrence of secondary structures and their effect on the detachment process of the LEV is still not fully understood, as described in Sect. 1; their emergence and effect on the instant when the LEV ceases to accumulate circulation will be investigated in detail in this section. Based on the observed comparability of results obtained at the two facilities, each evaluation will consider results from both facilities to extend the covered parameter range.

A combination of Lagrangian flow field analysis based on FTLE and Eulerian vortex characteristics is used for a detailed investigation of secondary structures and their effect on LEV characteristics, as discussed in Sect. 2.2. Figure 10 depicts FTLE flow fields in terms of attracting and repelling (backward and forward) FTLE ridges for different dimensionless time instants computed from velocity data obtained at the TUDA facility. All ridges that passed the $80 \%$ threshold are depicted at the same color level to allow topological structures to be identified more easily. Early in the downstroke at $t / T=0.15$, shown in Fig. 10a, the LEV is confined at the top and to the rear by a shellshaped repelling ridge (highlighted in red). At $t / T=0.175$ and for further time instants the confining ridge disappears, since its strength decreases. The leading edge shear layer feeding the LEV is demarcated in upstream direction by an attracting ridge (highlighted in green). In the downstream direction it is demarcated by an attracting ridge arising from the airfoil surface, forming a channel with the upstream demarcation line that curls up into the vortex. For $t / T=0.15$ and 0.175 there are no distinct flow structures visible at the leading edge, immediately above the airfoil. However from $t / T=0.175$ to 0.2 a region of fluid right above the airfoil surface at the leading edge emerges, which is isolated from the flow field by a repelling ridge. Fluid particles close to this ridge will depart from each other at future instances by becoming entrained into different topological structures, namely the main LEV and the secondary vortices, which are located close to the leading edge. The qualitative evolution of FTLE fields obtained from BUAA data was found to be in very good agreement regarding the observed topology.

\subsection{Secondary Structure Occurrence (SSO)}

In continuation of earlier efforts by Kissing et al. (2020), the occurrence of secondary structures is quantified with the approach introduced by Huang and Green (2015). This method identifies Lagrangian Coherent Structure (LCS) saddle points in the flow field by intersections of repelling and attracting ridges. The flow saddle point is indicated 


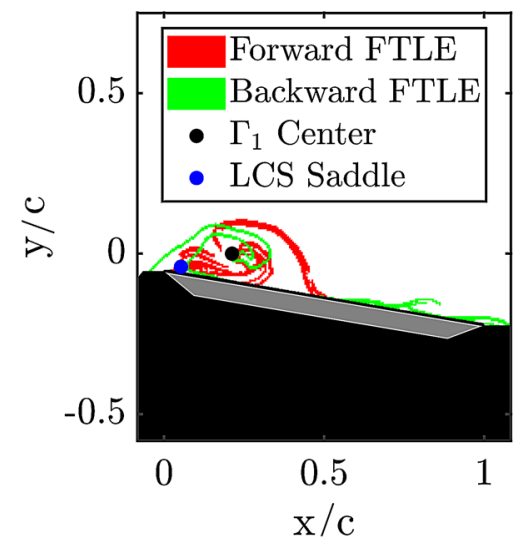

(a) $t / T=0.15$

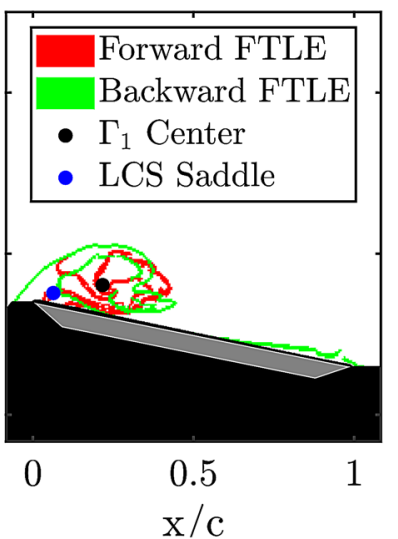

(b) $t / T=0.175$
Fig. 10 Flow field in terms of repelling and attracting ridges for different dimensionless time instants obtained from forward and backward FTLE computations for the common baseline case (ID 3 from TUDA). FTLE ridges are obtained using a $80 \%$ threshold of the

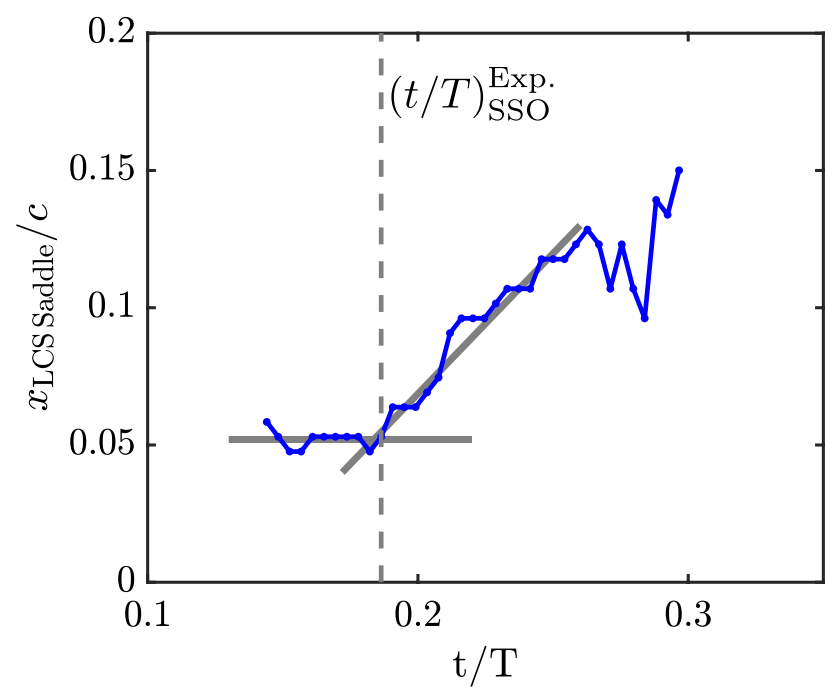

Fig. 11 Normalized streamwise LCS saddle position $x_{\text {LCS Saddle }} / c$ as a function of dimensionless time $t / T$. The position is extracted in a plate-fixed frame of reference. The convection of the LCS saddle at different phases is approximated linear and indicated by solid grey lines. The derived instant of secondary structure occurrence $(t / T)_{\text {SSO }}^{\text {Exp. }}$ is marked with a vertical dashed line

in Fig. 10 as a blue point. As soon as secondary structures arise and grow, the LCS saddle point moves downstream as a result of the increased area covered by the structures. Therefore, the streamwise LCS saddle location in a platefixed frame of reference is extracted to evaluate secondary structure growth as depicted for ID 3 case from TUDA in Fig. 11. The LCS saddle location was extracted by an automated identification of the first intersection of attracting and repelling ridges downstream of the leading edge.

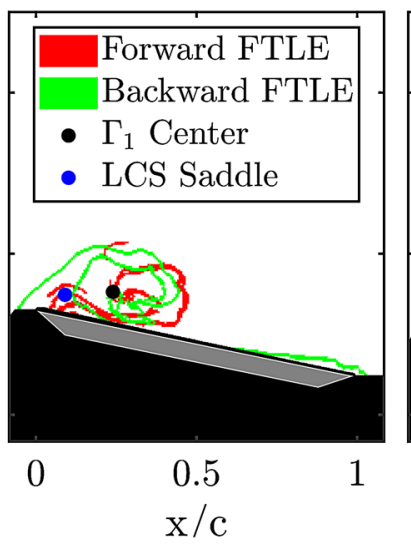

(c) $t / T=0.2$

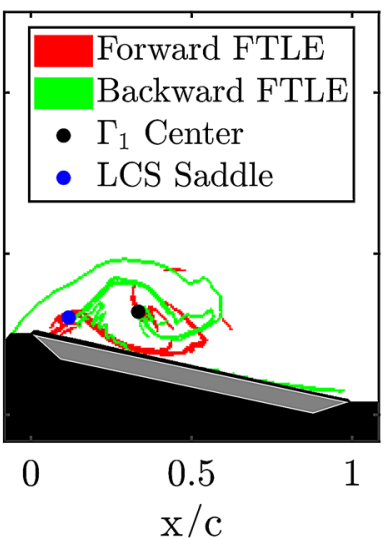

(d) $t / T=0.25$ respective maximum FTLE value in each frame. The airfoil is masked out in grey and the laser light shadow in black. The vortex center from $\Gamma_{1}$ criterion and the LCS saddle are marked as well

Only intersections below the height of the vortex center were considered.

After an initial phase where the LCS saddle remains close to the leading edge at about $x / c=0.05$, it starts to convect downstream shortly before $t / T=0.2$, as shown in Fig. 11, indicating an increase of the area covered by secondary structures and thus their growth. This evolution is in agreement with the observed secondary structure onset from FTLE fields in Fig. 10. To determine the instant of convection increase and thus secondary structure occurrence, the LCS saddle location is approximated to first order separately from $t / T=0.15$ to 0.18 and from 0.18 to 0.25 . The dimensionless time instant of secondary structure occurrence (SSO), denoted as $(t / T)_{\text {SSO }}^{\text {Exp. }}$, can be determined by the intersection of both convection slopes. It is marked in Fig. 11 by a vertical dashed line. The convection of the LCS saddle evaluated from FTLE fields obtained from BUAA for baseline case data was found to occur about $3 \%$ earlier than from TUDA with respect to the downstroke period, which is considered to be very good agreement. Subsequently, $(t / T)_{\text {SSO }}^{\text {Exp. }}$ was evaluated for all investigated cases.

The circulation of the LEV from vortex identification evaluations, discussed in respect to Fig. 6, was additionally used to compute the vortex Reynolds number $\operatorname{Re}_{v}=\Gamma_{\mathrm{LEV}} / v \pi$ proposed by Doligalski et al. (1994) at the determined secondary structure onset. The vortex Reynolds number represents the tendency of the boundary layer below the vortex on the airfoil surface to respond to the vortex induced pressure gradient. The viscous response of the boundary layer occurs concurrent with a high concentration of vorticity in the boundary layer and leads to an eruption of a fluid spire and subsequent formation of secondary structures. For low $\mathrm{Re}_{v}$, the vortex circulation and 
thus the induced velocity and pressure gradient on the airfoil surface are lower and the boundary layer response is suppressed. For higher $\mathrm{Re}_{v}$, the response is more likely triggered. For additional information on the phenomena, the reader is referred to Doligalski et al. (1994). $\mathrm{Re}_{v}$ is shown as

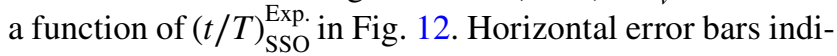
cate the error in identification of secondary structure onset from the convection of the LCS saddle and vertical bars the standard deviation of $\operatorname{Re}_{v}$ caused by the standard deviation of circulation at the determined secondary structure onset. Considering the secondary structure onset for cases with $\hat{\alpha}_{\text {eff }}$ $=30^{\circ}$ from TUDA, represented by the filled markers (ID 1 ; $2 ; 3 ; 7 ; 8$ ), it can be observed that secondary structures arise at earlier dimensionless times for lower $k$ (square markers) than for a higher ones (circle markers). The Strouhal number has a minor influence on the onset instant (different colours). Similar trends can be observed for $\hat{\alpha}_{\text {eff }}=20^{\circ}$ cases, indicated by the open markers (ID $4 ; 5 ; 6 ; 9 ; 10$ ), with a general temporal lag. The instants of secondary structure onset from BUAA agree well with the TUDA results, considering cases of the same parameters (ID 3 and 11 as well as ID 10 and 13). The observation of a minor influence of the Strouhal number is additionally confirmed by the ID 12 case agreement with ID 3 and 11 cases, since it denotes a case with parameters identical to them but with a higher St of 0.16 .

For parameters investigated in this study, secondary structures occur when the vortex Reynolds number reaches a threshold within a very narrow band for each setup, although the mean vortex Reynolds numbers $\overline{\operatorname{Re}}_{v}$ differ between both. For TUDA results, secondary structures occur for $\operatorname{Re}_{v}$ between 3500 and 3900 with $\overline{\operatorname{Re}}_{v}=$ 3,700 and a standard deviation $\sigma_{\mathrm{Re}_{v}}$ of about 150. At BUAA they arise for $\overline{\operatorname{Re}}_{v}=2900$ with a smaller $\sigma_{\mathrm{Re}_{v}}$ of about 90 . Reconsidering the comparison of circulation evolution for the common baseline case from Fig. 6, where an offset for earlier instants could be attributed to the inclusion of the leading edge shear layer for the TUDA evaluations, the higher $\mathrm{Re}_{v}$ from TUDA results can be expected and attributed to the vortex identification methodology.

Based on a known $\overline{\operatorname{Re}}_{v}$ threshold at the onset of secondary structures, which is in turn dependent on the LEV circulation, their onset could be predicted if the accumulation of circulation of the LEV were known. The observed $\overline{\operatorname{Re}}_{v}$ threshold indicates that secondary structures emerge at a certain circulation of the LEV. Their onset could be temporally determined via the instant where the LEV reaches a circulation threshold. This approach is considered a valuable prediction tool for future attempts to delay LEV detachment, which focus on secondary structure suppression. With the capability of predicting secondary structure emergence, their onset could be specifically delayed or suppressed. Wong and Rival (2015) derive an expression for the rate of circulation accumulation of the LEV, $\dot{\Gamma}_{\mathrm{LEV}}$, in which it is proportional to the square of the effective inflow velocity on the airfoil $u_{\text {eff }}$ :
Fig. 12 Vortex Reynolds number $\operatorname{Re}_{v}$ at secondary structure onset $(t / T)_{\text {SSO }}^{\text {Exp. Measurements at }}$ TUDA (ID 1-10) and measurements at BUAA (ID 11-13) are used to obtain separate mean vortex Reynolds numbers $\overline{R e}_{v}$ and their respective standard deviations $\sigma_{\mathrm{Re}_{\mathrm{v}}}$, indicated as dashed lines and grey patches; see Tables 1 and 2 for the respective parameter combinations of ID 1-13 and marker style schemes

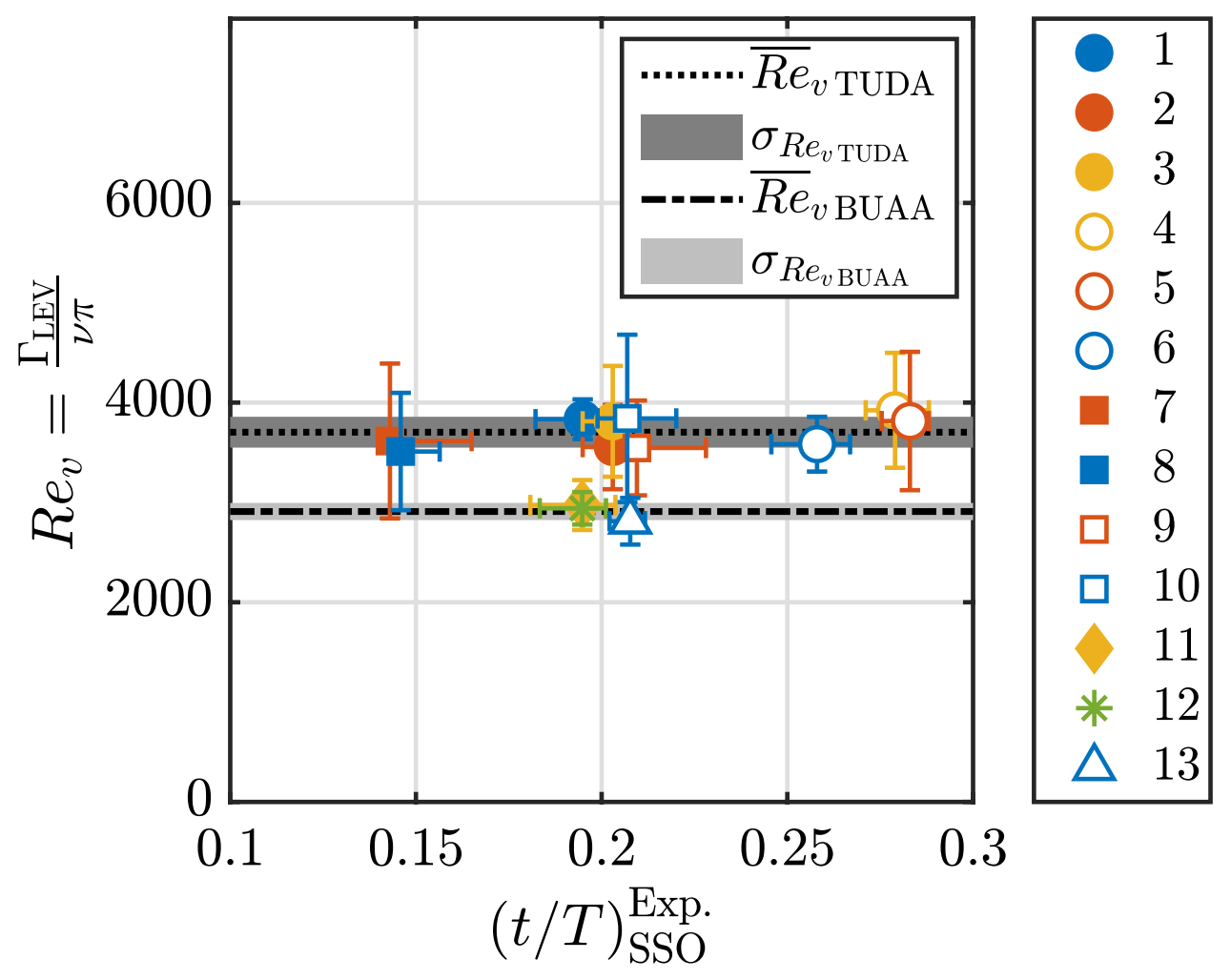


$\dot{\Gamma}_{\mathrm{LEV}} \propto u_{\mathrm{eff}}^{2}(t)$.

Their model builds upon an analytical expression for the mass flux into the LEV, derived by Wong et al. (2013) based on the vorticity transport equation. With an approximation of the circulation flux per mass flux from a path integral around the shear layer, the circulation flux into the LEV is derived. Their model was found to capture $\Gamma_{\mathrm{LEV}}$, experimentally obtained via vortex identification, precisely for cases where the LEV forms very early in the downstroke.

During the comparison of the circulation evolution predicted by the aforementioned model and measured evolution, significant deviations were encountered for several cases. In these cases the LEV was found to emerge delayed in respect with the motion start, caused by a delayed rollup of the leading edge shear layer. This led to a temporal offset of the measured LEV circulation with respect to the predicted value, although the slope of circulation accumulation was still in very good agreement. To account for the observed delayed LEV formation, Mulleners and Raffel (2012) developed a scaling for the temporal LEV formation lag with respect to the motion start $t_{\mathrm{LEV} \text { onset }}^{\text {Exp. }}$ which is tested as a potential solution to adapt the aforementioned circulation flux model. It is based on the assumption that the rate of change of the inflow angle on the airfoil during the period when the inflow angle exceeds the static stall angle is responsible for the temporal lag of LEV formation. Figure 13 shows the correlation between the rate of change of the effective inflow angle during this period $\dot{\alpha}_{\text {eff, ss }}$ and the LEV onset delay $t_{\mathrm{LEV} \text { onset }}^{\text {Exp. }}$, determined by visual inspection of FTLE fields. By comparing the observed LEV emergence

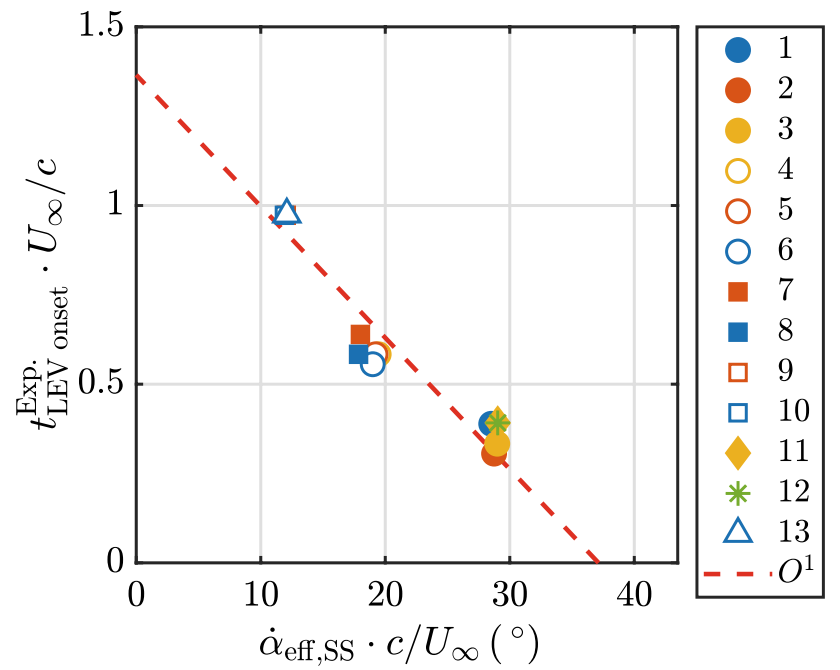

Fig. 13 LEV onset delay $t_{\mathrm{LEV}}^{\text {Exp }}$ onset as a function of the rate of change of the effective inflow angle on the airfoil during static stall angle $\dot{\alpha}_{\text {eff,Ss }}$. A linear approximation of the scaling is indicated as dashed red line; see Tables 1 and 2 for the respective parameter combinations of ID 1-13 and marker style schemes from TUDA and BUAA evaluations with a linear approximation according to

$t_{\mathrm{LEV} \text { onset }}^{\text {Exp. }} \cdot \frac{U_{\infty}}{c}=a \cdot \dot{\alpha}_{\mathrm{eff}, \mathrm{SS}} \cdot \frac{c}{U_{\infty}}+b$,

indicated by the dashed red line, good agreement in terms of a linear scaling by $\dot{\alpha}_{\text {eff,SS }}$ can be observed. $a$ and $b$ are empirical parameters determined from reference measurements.

Accordingly, the circulation flux model by Wong and Rival (2015) is adapted to account for the delayed vortex formation. This is done by offsetting temporal information used to integrate $\dot{\Gamma}_{\mathrm{LEV}}$ from Eq. (1) according to the approximated $t_{\mathrm{LEV} \text { onset }}^{\text {Exp. }}$

$\Gamma_{\mathrm{LEV}}=\int u_{\mathrm{eff}}^{2} \mathrm{~d}\left(t-t_{\mathrm{LEV} \text { onset }}^{\text {Exp. }}\right)$.

By using separate circulation threshold values, derived from $\overline{\operatorname{Re}}_{v}$ thresholds for TUDA and BUAA, the dimensionless time instant of expected secondary structure onset $(t / T)_{\mathrm{SSO}}^{\text {Theor. }}$ is obtained from the adapted circulation flux model. It is compared to the experimentally observed secondary struc-

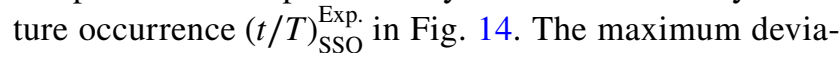
tion between the predicted and measured secondary structure occurrence $\Delta(t / T)$ is 0.0248 (equivalent to $4.9 \%$ of the downstroke period), which is considered as a reasonably accurate prediction.

With the introduced model for secondary structures, it is possible to compute their onset based on kinematic parameters. Therefore, the effective inflow velocity, determined by the inflow and plunging velocity, and the pitching rate

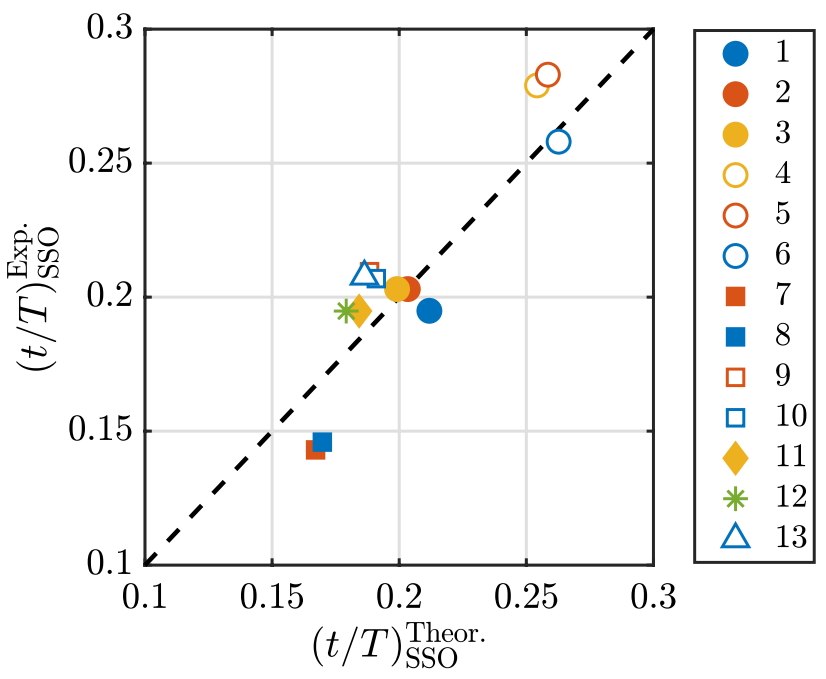

Fig. 14 Modelling secondary structure occurrence $(t / T)_{\text {SSO }}^{\text {Theor. }}$ based on a vortex Reynolds number threshold, taking the delay of LEV formation in circulation approximation into account; see Tables 1 and 2 for the respective parameter combinations of ID 1-13 and marker style schemes 
evolution have to be known. The only necessary empirical input is the scaling parameter for the delay in LEV formation $t_{\mathrm{LEV} \text { onset }}^{\text {Exp. }}$. This can be obtained using a limited number of reference measurements.

\subsection{Secondary structure effect on LEV detachment}

The role of the effective inflow angle amplitude on the airfoil in the LEV detachment process has not been considered in detachment mechanisms proposed in literature (cf. Sect. 1). Therefore, the effect of secondary structures in the detachment process is discussed in the following by consideration of different effective angle of attack amplitudes. This discussion will focus on secondary structure effects on the termination of circulation accumulation of the LEV to clarify their role as a trigger of secondary topological structures. It should be noted that the LEV detachment can also be defined in terms of the lift force decrease, which however, has not been measured in this study.

To identify parameter sets where circulation accumulation of the LEV stops as a direct consequence of secondary structure emergence, the concurrency of both events is compared. This is done with the aid of the dimensionless time lag between secondary structure onset $\left((t / T)_{\mathrm{SSO}}^{\text {Exp. }}\right)$ and maximum LEV circulation $\left((t / T)^{\Gamma_{\mathrm{LEV}} \max }\right)$, denoted as $\Delta(t / T)_{\mathrm{SSO}}^{\Gamma_{\mathrm{LEV}} \max }$. It is shown in Fig. 15 as a function of the effective inflow angle amplitude on the airfoil $\hat{\alpha}_{\text {eff }}$. The instant of secondary structure onset is determined by topological FTLE field evaluations according to the methodology introduced along the discussion of Fig. 11 and the instant

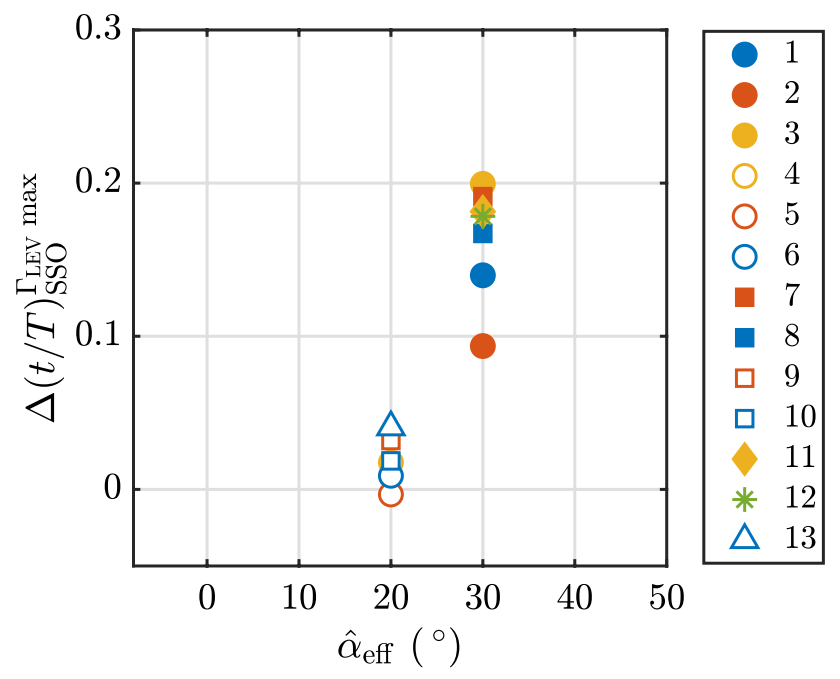

Fig. 15 Dimensionless time lag between the onset of secondary structures and the instant of maximum LEV circulation $\Delta(t / T)_{\mathrm{SSO}}^{\Gamma_{\mathrm{LEV}}}{ }^{\max }$ to evaluate the concurrency of both events, shown over of the effective inflow angle amplitude on the airfoil $\hat{\alpha}_{\text {eff }}$; see Tables 1 and 2 for the respective parameter combinations of ID 1-13 and marker style schemes of LEV peak circulation from vortex identification results, indicated by the dashed lines in Fig. 6. For parameter sets with $\hat{\alpha}_{\text {eff }}=20^{\circ}$ a small time lag can be observed. On the other hand the time lag is larger for cases with $\hat{\alpha}_{\text {eff }}=30^{\circ}$.

Although $\hat{\alpha}_{\text {eff }}$ seems to allow for a classification whether secondary structure occurrence causes circulation accumulation termination as a more direct consequence, it does not scale $\Delta(t / T)_{\mathrm{SSCO}}^{\Gamma_{\mathrm{LEV}} \max }$ directly nor solely, as evident from different $\Delta(t / T)_{\mathrm{SSO}}^{T_{\mathrm{LEV}}^{\max }}$ for the same $\hat{\alpha}_{\text {eff }}$. Additional scalings have been tested on results presented in Fig. 15. A correlation of $\dot{\alpha}_{\text {eff, SS }}$ with $\Delta(t / T)_{\mathrm{SSO}}^{\Gamma_{\mathrm{LEV}} \max }$ did not exhibit correlation of both quantities. Convective normalization of the time lag has also been considered, but did not exhibit clear correlations. Recall that such a convective scaling might allow modelling of secondary structure onset via circulation accumulation and the $\operatorname{Re}_{v}$ threshold, but does not directly determine effects of secondary structures on LEV detachment. For all cases with $\hat{\alpha}_{\text {eff }}=20^{\circ}$, fluid recirculation around the trailing edge occurred significantly after the LEV reaches its peak circulation.

These observations suggest that for lower effective angle of attack amplitudes of $20^{\circ}$, the emergence of secondary structures causes the LEV to stop the accumulation of circulation independent of fluid recirculation around the trailing edge. This is in accordance with the detachment mechanism defined by Widmann and Tropea (2015). On the other hand, the termination of circulation accumulation of the LEV occurs coincident with fluid recirculation in the common baseline case with $\hat{\alpha}_{\text {eff }}=30^{\circ}$, discussed in section 3. As it can be seen in Fig. 12, secondary structures occur already at $t / T=0.2$, but do not cause the LEV to stop accumulating circulation as a direct consequence in this case. This suggests a detachment in accordance with Rival et al. (2014).

To validate if fluid recirculation around the trailing edge causes the LEV to stop accumulation of circulation for all cases with $\hat{\alpha}_{\text {eff }}=30^{\circ}$, they were investigated by means of the tangential velocity plot discussed in respect to Fig. 9. The instant of fluid recirculation around the trailing edge and maximum LEV circulation coincide or recirculation occurs slightly before maximum circulation for all cases. This confirms that the LEV detaches in accordance with the mechanism described by Rival et al. (2014) in cases where $\hat{\alpha}_{\text {eff }}$ is $30^{\circ}$. The change of the prevalent detachment mechanism for different $\hat{\alpha}_{\text {eff }}$ but the same dimensionless parameters highlights its role as an important parameter influencing vortex detachment. Additionally, the LEV stops accumulating circulation as a more direct consequence of secondary structure occurrence for cases with a lower $\hat{\alpha}_{\text {eff }}$. Both facts clearly indicate that $\hat{\alpha}_{\text {eff }}$ has to be taken into account in models that aim to distinguish between detachment mechanisms like the covering ratio $\kappa$. The mechanism, which causes the LEV to stop accumulating circulation after secondary structure occurrence will be investigated further below. 
A potential scenario explaining how secondary structures cause the LEV to stop accumulating circulation is an interaction of the shear layer fluid from the leading edge of the airfoil with secondary structures, which takes place before the shear layer fluid reaches the LEV. An interaction of the negatively signed vortical fluid from the shear layer with the positively signed of secondary structures would presumably result in cross-annihilation of vorticity, as described by Wojcik and Buchholz (2014). Additionally, the shear layer of high velocity could push secondary structures and the main LEV downstream if a low shear layer angle directs fluid directly into those structures. Likewise, secondary structures could guide the shear layer curvature in an upward or downward direction such that it no longer reaches the main LEV.

The geometric factors determining whether an interaction of any kind is enabled are the evolution of position and size of secondary structures in combination with the shear layer angle. As soon as secondary structures arise, they will grow and consequently cover a larger area. If the shear layer angle does not increase further from the instant of secondary structure onset, an interaction of both due to growing secondary structures is a direct consequence. For lower peak inflow angles on the airfoil the shear layer angle is assumed to reach lower peak values and an interaction of secondary structures with the shear layer occurs earlier. This could explain the observed concurrency of secondary structure onset and the termination of circulation accumulation of the LEV for cases where $\hat{\alpha}_{\text {eff }}$ is $20^{\circ}$ as shown in Fig. 15.

To test this hypothesis, the influence of the shear layer angle after separation from the leading edge $\alpha_{\mathrm{SL}}$ will be investigated as a potential factor causing the simultaneous cessation of circulation accumulation and onset of secondary structures for $\hat{\alpha}_{\text {eff }}=20^{\circ}$. The methodology used to extract $\alpha_{\mathrm{SL}}$ from vorticity fields is based on the fact that the leading edge shear layer is characterized by very high vorticity values. This methodology is illustrated exemplary for the ID 8 Case at $t / T=0.26$ in Fig. 16. Maximum vorticity values, denoted as $\omega_{\max }$ and indicated as orange dots in Fig. 16, are extracted in a square, plate-fixed region of interest (ROI) that spans from the leading edge downstream up to the region of secondary structures. The final $\alpha_{\mathrm{SL}}$ evolution is obtained from the angle between a first order fitted line $\left(O^{1}\right)$ of the extracted maximum value locations, indicated by a red line in Fig. 16, and the airfoil surface. To exclude large fluctuations of the shear layer angle, its evolution is smoothed using a second order Savitzky-Golay filter of 15 frames width (corresponding to $\Delta(t / T)=0.1$ ). After extraction of the angle from single runs, it is ensemble averaged for each parameter set. An exemplary evolution of $\alpha_{\mathrm{SL}}$ for the ID 8 case from TUDA is depicted in Fig. 17, where the direct ensemble averaged angle evolution is shown in grey and the smoothed in blue with error bars, indicating the standard deviation between single runs.

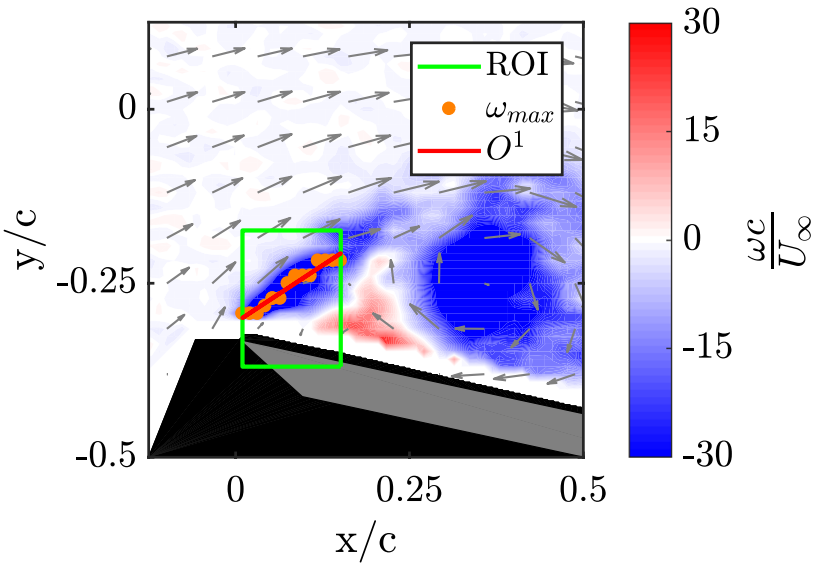

Fig. 16 Extraction methodology of the shear layer angle after separation from the leading edge with respect to the airfoil surface $\alpha_{\mathrm{SL}}$. The normalized vorticity field around the leading edge region is depicted at $t / T=0.26$ from ID 8 case. Highest vorticity values $\left(\omega_{\max }\right)$ within the region of interest (ROI) are identified to obtain a first order approximated line $\left(O^{1}\right)$. The inflow is from the left, the airfoil is masked out in grey and the laser light shadow in black

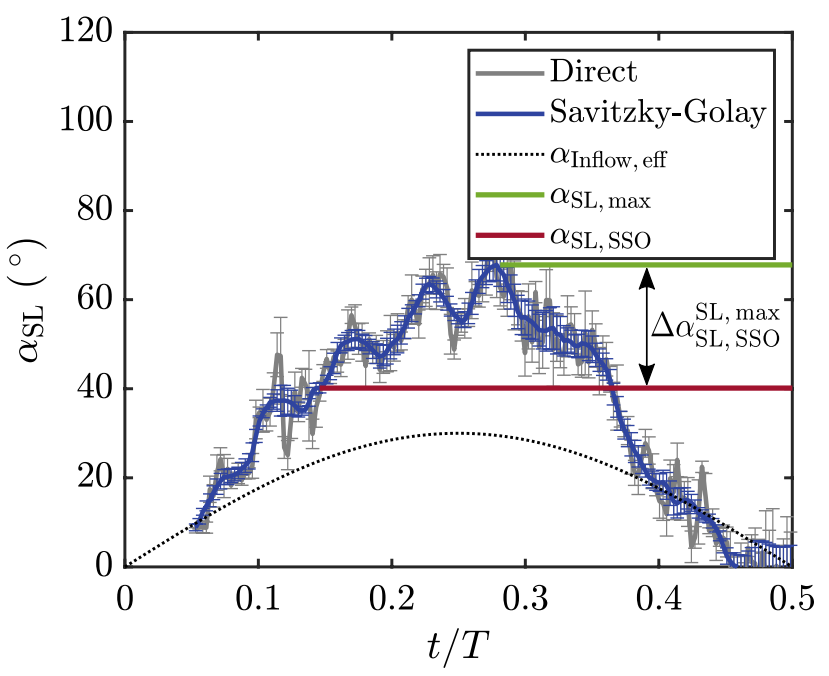

Fig. 17 Ensemble averaged evolution of the extracted shear layer angle after separation from the leading edge $\alpha_{\mathrm{SL}}$ over dimensionless time $t / T$ for the ID 8 case from TUDA. Error bars represent the standard deviation of single run ensemble averaging. The shear layer angle during secondary structure onset $\alpha_{\mathrm{SL}, \text { SSO }}$ and the overall maximum

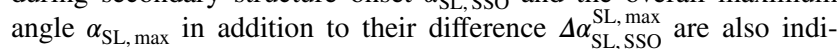
cated. The effective inflow angle on the airfoil $\alpha_{\text {Inflow, eff }}$ is added for orientation; see Tables 1 and 2 for the respective parameter combinations of ID 1-13 and marker style schemes

Two geometric factors determine where an interaction between secondary structures and the leading edge shear layer is initiated. The first factor is the size of secondary structures and the second is the shear layer angle evolution after secondary structure emergence. Secondary structures will grow after their emergence and if the shear layer angle 
does not increase further during this period, an interaction is initiated. So the difference between the shear layer angle at secondary structure onset $\alpha_{\mathrm{SL} \text {, SSO }}$ and the maximum shear layer angle $\alpha_{\mathrm{SL}, \max }$ is considered as a measure where interaction likely occurs. This difference is denoted as $\Delta \alpha_{\mathrm{SL}, \mathrm{SSO}}^{\mathrm{SL}}$ and highlighted in Fig. 17 by a double-sided arrow. For larger values of $\Delta \alpha_{\mathrm{SL}, \mathrm{SSO}}^{\mathrm{SL}}$ the shear layer angle increases more after secondary structure onset and an interaction is unlikely to take place immediately, while it is likely to occur directly if the angle does not increase further. Fig. 18 shows the concurrency of the emergence of secondary structures and the peak circulation of the LEV as a function of $\Delta \alpha_{\mathrm{SL} \text {, SSP }}^{\mathrm{SL} \text {. In }}$ cases with $\hat{\alpha}_{\text {eff }}=20^{\circ}$, indicated by open symbols, $\Delta \alpha_{\mathrm{SL}, \mathrm{SSO}}^{\mathrm{S}}$ is generally lower, so the shear layer angle at the leading edge increases less from the instant of secondary structure onset before reaching its maximum. This trend occurs concurrent with lower $\Delta(t / T)_{\mathrm{SSO}}^{\Gamma_{\mathrm{LEV}} \max }$ values, indicating a circulation accumulation stop of the vortex as a more direct consequence of secondary structure emergence. In contrast, the shear layer angle increases significantly after secondary structure emergence before reaching its maximum for $\hat{\alpha}_{\text {eff }}=$ $30^{\circ}$ (filled markers). As discussed above, an interaction of secondary structures and the shear layer is assumed to be delayed for cases where the shear layer continues to increase after secondary structure emergence. For these conditions, secondary structures have sufficient space to grow without an interaction with the shear layer. The observations indicate that the interaction of the leading edge shear layer with secondary structures causes the LEV to stop accumulation of circulation as a direct consequence in cases with $\hat{\alpha}_{\text {eff }}=20^{\circ}$. It also highlights the interaction as the main cause for the

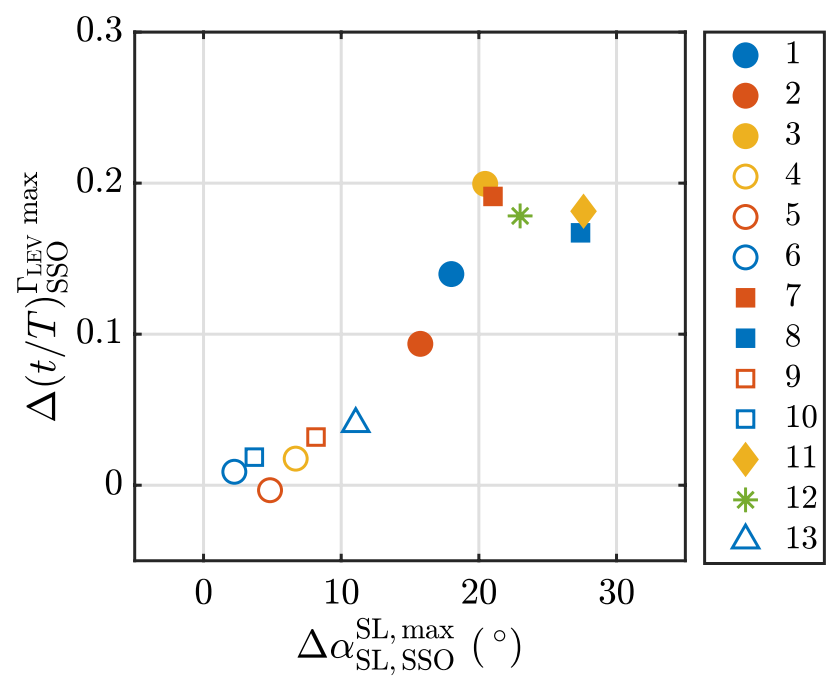

Fig. 18 Correlation of the temporal concurrency between circulation accumulation stop of the LEV and secondary structure occurrence $\Delta(t / T)_{\mathrm{SSO}}^{\Gamma_{\mathrm{LEV}} \max }$ with the shear layer angle difference at secondary structure onset and the maximum angle $\Delta \alpha_{\mathrm{SL}, \mathrm{SSO}}^{\mathrm{SL}}$; see Table 1 and 2 for the respective parameter combinations of ID 1-13 change of the prevalent detachment mechanism observed in respect to Fig. 15 for cases with different $\hat{\alpha}_{\text {eff }}$ but the same key parameters.

For either case in Fig. $18, \Delta(t / T)_{\mathrm{SSO}}^{\Gamma_{\mathrm{LEV}} \max }$ and $\Delta \alpha_{\mathrm{SL}, \mathrm{SSO}}^{\mathrm{SL}, \max }$ correlate. Under the assumption, that the onset of interaction is determined by the shear layer angle increase after secondary structure occurrence, this shows that an interaction leads to the termination of circulation accumulation of the LEV, independent of recirculation.

\section{Conclusions}

In this study an approach to establish comparability of flow fields and LEV characteristics on a pitching and plunging flat plate obtained from facilities working with air and water as media is developed and validated. An order of magnitude difference in viscosity of the media extends the attainable parameter space in terms of dimensionless parameters.

The definition of a common baseline case was found to require careful consideration of the viable parameter range due to inherently different time scales of the airfoil motion caused by the difference in viscosity. Flow fields and topological evolution agreed well in air and water, but the LEV circulation differed. An intermittent inclusion of the leading edge shear layer into the LEV domain was identified as the source for deviations of the computed circulation. This highlights that small deviations in velocity information can affect circulation evaluations via the identified vortex domain used for circulation computation, even when using the same correlation algorithm. As already stated in Sect. 2, data sets of baseline cases from TUDA and BUAA are also available online as reference cases (http://dx.doi.org/10.25534/tudat alib-168).

An investigation of secondary structures ahead of the LEV with the aid of a Lagrangian flow field analysis by FTLE ridges allowed a precise identification of their onset and effect on the LEV detachment process. The combination of both facilities enabled investigations of secondary structures over an extended parameter range, including variations of the reduced frequency, the Strouhal number and the effective angle of attack amplitude.

Secondary structures were found to emerge at similar vortex Reynolds numbers, computed from LEV circulation, at each setup and for all investigated parameters. Secondary structure occurrence, which is triggered by a viscous response of the boundary layer below the airfoil, is thus governed by the vortex circulation.

With the aid of existing circulation flux models from literature and their adaptation to account for a delayed LEV formation, a model to predict the temporal occurrence of secondary structures was developed and found to be in agreement with the observed onset. 
For cases with a lower effective angle of attack amplitude, the LEV was found to cease accumulate circulation in close temporal correlation with secondary structure emergence and prior to fluid recirculation around the trailing edge. For cases with the same dimensionless parameters but a higher effective angle of attack amplitude, the vortex stopped accumulating circulation concurrent with fluid recirculation around the trailing edge. This highlights the effective angle of attack as an important parameter determining the prevalent LEV detachment mechanism, which should be considered for future LEV detachment investigations.

The temporal concurrence of secondary structure onset and the circulation accumulation stop was found to closely correlate with the leading edge shear layer angle increase after secondary structure onset. It is hypothesized that the interaction of secondary structures with the shear layer from the leading edge causes the observed correlation. This interaction occurs sooner after secondary structure occurrence for lower effective angles of attack, since the shear layer angle increases less after secondary structure occurrence. An in-depth study of the effect of secondary structure growth on the shear layer angle evolution by means of pressure field analysis is foreseen to provide sufficient information on their exact cause-effect relationship. Nevertheless, the observed correlation allows to infer, that the interaction of secondary structures with the shear layer causes the observed difference in LEV detachment for different effective angle of attack amplitudes.

Cessation of circulation accumulation of the LEV due to the occurrence of secondary structures implies that a suppression of their onset or growth is a promising approach for future flow control strategies targeting a prolonged LEV growth phase. The timing of secondary structure suppression can be optimized using the developed model for secondary structure onset. Alternatively, the interaction of the shear layer with secondary structures could be prevented/delayed by directing the shear layer upward. Both approaches are assumed to be more effective for cases with a lower effective angle of attack amplitude due the direct stop of circulation accumulation of the LEV as a consequence of interaction in those cases.

Acknowledgements The authors wish to acknowledge financial support of the Sino-German Center and the Deutsche Forschungsgemeinschaft through the project TR 194/55-1 and GZ-1280: "Flow Control for Unsteady Aerodynamics of Pitching/Plunging Airfoils". The authors would also like to extend their appreciation to the workshop staff in Darmstadt for their assistance and professional support for these experiments.

\section{Funding Open Access funding provided by Projekt DEAL.}

Open Access This article is licensed under a Creative Commons Attribution 4.0 International License, which permits use, sharing, adaptation, distribution and reproduction in any medium or format, as long as you give appropriate credit to the original author(s) and the source, provide a link to the Creative Commons licence, and indicate if changes were made. The images or other third party material in this article are included in the article's Creative Commons licence, unless indicated otherwise in a credit line to the material. If material is not included in the article's Creative Commons licence and your intended use is not permitted by statutory regulation or exceeds the permitted use, you will need to obtain permission directly from the copyright holder. To view a copy of this licence, visit http://creativecommons.org/licenses/by/4.0/.

\section{References}

Akkala JM, Buchholz JHJ (2017) Vorticity transport mechanisms governing the development of leading-edge vortices. J Fluid Mech 829:512-537. https://doi.org/10.1017/jfm.2017.559

Benedict LH, Gould RD (1996) Towards better uncertainty estimates for turbulence statistics. Exp Fluids 22:129-136. https://doi. org/10.1007/s003480050030

Carr LW (1988) Progress in analysis and prediction of dynamic stall. J Aircr 25:6-17. https://doi.org/10.2514/3.45534

Champagnat F, Plyer A, Le Besnerais G, Leclaire B, Davoust S, Le Sant Y (2011) Fast and accurate piv computation using highly parallel iterative correlation maximization. Exp Fluids 50:1169. https://doi.org/10.1007/s00348-011-1054-X

de Croon G, Perçin M, Remes B, Ruijsink R, de Wagter C (2015) The DelFly: Design, aerodynamics, and artificial intelligence of a flapping wing robot. Springer, Dordrecht

Doligalski TL, Smith CR, Walker JDA (1994) Vortex interactions with walls. Annu Rev Fluid Mech 26:573-616. https://doi. org/10.1146/annurev.fl.26.010194.003041

Eldredge JD, Jones AR (2019) Leading-edge vortices: mechanics and modeling. Annu Rev Fluid Mech 51:75-104. https://doi. org/10.1146/annurev-fluid-010518-040334

Ellington CP (1984) The aerodynamics of hovering insect flight. iv. aeorodynamic mechanisms. Philos Trans R Soc B Biol Sci 305:79-113. https://doi.org/10.1098/rstb.1984.0052

Ellington CP, van den Berg C, Willmott AP, Thomas ALR (1996) Leading-edge vortices in insect flight. Nature 384:626-630. https://doi.org/10.1038/384626a0

Eslam Panah A, Akkala JM, Buchholz JHJ (2015) Vorticity transport and the leading-edge vortex of a plunging airfoil. Exp Fluids 56:155. https://doi.org/10.1007/s00348-015-2014-7

Foss JF (2004) Surface selections and topological constraint evaluations for flow field analyses. Exp Fluids 37:883-898. https:// doi.org/10.1007/s00348-004-0877-0

Graftieaux L, Michard M, Grosjean N (2001) Combining piv, pod and vortex identification algorithms for the study of unsteady turbulent swirling flows. Meas Sci Technol 12:1422-1429. https ://doi.org/10.1088/0957-0233/12/9/307

Haller G (2002) Lagrangian coherent structures from approximate velocity data. Phys Fluids 14:1851-1861. https://doi. org/10.1063/1.1477449

Huang Y, Green MA (2015) Detection and tracking of vortex phenomena using Lagrangian coherent structures. Exp Fluids 56:41. https://doi.org/10.1007/s00348-015-2001-z

Hunt JC, Wray AA, Moin P (1988) Eddies, streams, and convergence zones in turbulent flows. In: 2. Proceedings of the (1988) Summer Program Studying Turbulence Using Numerical Simulation Databases. United States, Stanford

Jones AR, Babinsky H (2010) Unsteady lift generation on rotating wings at low reynolds numbers. J Aircr 47:1013-1021. https:// doi.org/10.2514/1.46649

Jones K, Platzer M (2000) Flapping-wing propulsion for a micro air vehicle. In: Proceedings of the 38th Aerospace Sciences Meeting and Exhibit, American Institute of 
Aeronautics and Astronautics, Reno, United States, https://doi. org/10.2514/6.2000-897

Kissing J, Kriegseis J, Tropea C (2020) On the role of secondary structures during leading edge vortex lift off and detachment on a pitching and plunging flat plate. New results in numerical and experimental fluid mechanics XII. Springer, Berlin, pp 204-213. https://doi.org/10.1007/978-3-030-25253-3_20

Mulleners K, Raffel M (2012) The onset of dynamic stall revisited. Exp Fluids 52:779-793. https://doi.org/10.1007/s0034 8-011-1118-y

Nudds RL, Taylor GK, Thomas ALR (2004) Tuning of strouhal number for high propulsive efficiency accurately predicts how wingbeat frequency and stroke amplitude relate and scale with size and flight speed in birds. Proc Biol Sci 271:2071-2076. https://doi. org/10.1098/rspb.2004.2838

Peng J, Dabiri JO (2009) Transport of inertial particles by lagrangian coherent structures: Application to predator-prey interaction in jellyfish feeding. J Fluid Mech 623:75-84. https://doi.org/10.1017/ S0022112008005089

Perry AE, Chong MS (1987) A description of eddying motions and flow patterns using critical-point concepts. Annu Rev Fluid Mech 19:125-155. https://doi.org/10.1146/annurev.fl.19.010187.001013

Raffel M, Kompenhans J, Wereley ST, Willert CE (2007) Particle image velocimetry: a practical guide. Springer, Berlin

Rival DE, Kriegseis J, Schaub P, Widmann A, Tropea C (2014) Characteristic length scales for vortex detachment on plunging profiles with varying leading-edge geometry. Exp Fluids 55(037):103. https://doi.org/10.1007/s00348-013-1660-x

Sattari P, Rival DE, Martinuzzi RJ, Tropea C (2012) Growth and separation of a start-up vortex from a two-dimensional shear layer. Phys Fluids 24:107102. https://doi.org/10.1063/1.4758793
Shadden SC, Dabiri JO, Marsden JE (2006) Lagrangian analysis of fluid transport in empirical vortex ring flows. Phys Fluids 18:047105. https://doi.org/10.1063/1.2189885

Triantafyllou GS, Triantafyllou MS, Grosenbaugh MA (1993) Optimal thrust development in oscillating foils with application to fish propulsion. J Fluids Struct 7:205-224. https://doi.org/10.1006/ jfls.1993.1012

Westerweel J, Scarano F (2005) Universal outlier detection for piv data. Exp Fluids 39:1096-1100. https://doi.org/10.1007/s0034 8-005-0016-6

Widmann A, Tropea C (2015) Parameters influencing vortex growth and detachment on unsteady aerodynamic profiles. J Fluid Mech 773:432-459. https://doi.org/10.1017/jfm.2015.259

Wojcik CJ, Buchholz JHJ (2014) Vorticity transport in the leading-edge vortex on a rotating blade. J Fluid Mech 743:249-261. https://doi. org/10.1017/jfm.2014.18

Wong JG, Rival DE (2015) Determining the relative stability of leading-edge vortices on nominally two-dimensional flapping profiles. J Fluid Mech 766:611-625. https://doi.org/10.1017/jfm.2015.39

Wong JG, Kriegseis J, Rival DE (2013) An investigation into vortex growth and stabilization for two-dimensional plunging and flapping plates with varying sweep. J Fluids Struct 43:231-243. https ://doi.org/10.1016/j.jfluidstructs.2013.09.010

Zhou J, Adrian RJ, Balachandar S, Kendall T (1999) Mechanisms for generating coherent packets of hairpin vortices in channel flow. J Fluid Mech 387:353-396. https://doi.org/10.1017/S002211209 900467X

Publisher's Note Springer Nature remains neutral with regard to jurisdictional claims in published maps and institutional affiliations.

\section{Affiliations}

\section{Johannes Kissing ${ }^{1}\left[\right.$ Jochen Kriegseis $^{2} \cdot$ Zhenyao $\mathrm{Li}^{3} \cdot$ Lihao Feng $^{3} \cdot$ Jeanette Hussong ${ }^{1} \cdot$ Cameron Tropea $^{1}$}

1 Institute of Fluid Mechanics and Aerodynamics, Technische Universität Darmstadt, Flughafenstrasse 19, 64347 Griesheim, Germany

2 Institute of Fluid Mechanics, Karlsruhe Institute of Technology, Kaiserstr. 10, 76131 Karlsruhe, Germany
3 Institute of Fluid Mechanics, Beihang University, No. 37 Xueyuan Road, Haidian District, Beijing 100191, People's Republic of China 